\title{
Butterflies (Lepidoptera: Papilionoidea and Hesperoidea) and other protected fauna of Jones Estate, a dying watershed in the Kumaon Himalaya, Uttarakhand, India
}

\author{
Peter Smetacek
}

Butterfly Research Centre, The Retreat, Jones Estate, Bhimtal, Uttarakhand 263136, India

Email: petersmetacek@rediffmail.com

Date of publication (online): 26 August 2012 Date of publication (print): 26 August 2012 ISSN $0974-7907$ (online) | 0974-7893 (print)

Editor: Rudi Mattoni

\section{Manuscript details:}

Ms \# 03020

Received 25 November 2011

Final received 02 February 2012

Finally accepted 15 July 2012

Citation: Smetacek, P. (2012). Butterflies (Lepidoptera: Papilionoidea and Hesperoidea) and other protected fauna of Jones Estate, a dying watershed in the Kumaon Himalaya, Uttarakhand, India. Journal of Threatened Taxa 4(9): 2857-2874.

Copyright: (c) Peter Smetacek 2012. Creative Commons Attribution 3.0 Unported License. JoTT allows unrestricted use of this article in any medium for non-profit purposes, reproduction and distribution by providing adequate credit to the authors and the source of publication.

Author Details: Peter SMetACEK is an authority on Indian Lepidoptera and has pioneered the use of insect communities as bio-indicators of climatic change and ground water.

Acknowledgements: I am grateful to my late father, Fred Smetacek Sr.; to the Times Fellowship Council, New Delhi, for a Fellowship to study Indian rivers in 1992 and to the Rufford Smal Grant Foundation, U.K., for funding the work on Lepidoptera and Himalayan forest ecosystems between 2006 and the present study via a series of grants. I am indebted to Rudi Mattoni, Argentina for encouragement to write this and valuable suggestions on an earlier draft and drawing my attention to the format developed by him and used in Table 1 and to Zdenek Faltynek Fric, Ceske Budejovice in the Czech Republic, for valuable taxonomic comments on Table 1. Also, am indebted to the anonymous referees whose recommendations considerably improved the paper and to my children, Kanika and Pius, who spent many hours sorting through note books, loose leaf lists, books and specimens to compile the enormous amount of data that went into the making of Table 1.
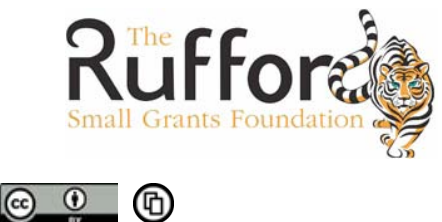

OPEN ACCESS | FREE DOWNLOAD
Abstract: Two hundred and forty three species of butterflies recorded from Jones Estate, Uttarakhand between 1951 and 2010 are reported. The ongoing rapid urbanization of Jones Estate micro-watershed will destroy the habitat of 49 species of wildlife protected under Indian law, as well as several species of narrow endemic moths and butterflies. The only known Indian habitat for the butterfly Lister's Hairstreak Pamela dudgeoni will be destroyed. The effect on the water flow of both the Bhimtal and Sattal lake systems will clearly be adverse, as is evident from the drying up of Kua Tal and the reduced flow of perennial water springs during the dry season on the Estate. The undoubtedly negative effect of urbanization on these valuable fresh water resources will be irreversible in the long term. The trend can be reversed by extending protection to Jones Estate by re-declaring it a Green Belt of Bhimtal and by banning construction in the catchment area of Bhimtal lake, as has been done in Nainital and Mussoorie, both in Uttarakhand.

Keywords: Bhimtal, drinking water, drying lakes, freshwater resources, Green Belt, Indian Wildlife (Protection) Act 1972, Pamela dudgeoni.

Hindi Abstract: सन् 1951 और 2010 के बीच जौंस स्टेट उत्तराखण्ड में संकलित 243 प्रजाति की तितलियों का वर्णन दिया गया है। वर्तमान में जौस स्टेट सूक्ष्म जलागम में तेजी स`चल रही षहरीकरण की प्रक्रिया से भारतीय कानून के अन्तर्गत संरक्षित 49 । वन जीव की प्रजातियां तथा कई विषेश पतंगे और तितलियों की प्रजाति (जो केवल जौंस स्टेट में पाई जाती हैं) के आवास नश्ट हो जायेंगे। इसमें लिस्ट्र्स हैयर स्ट्रीक तितली का भारत में एक मात्र निवास नश्ट हो जायेगा। भीमताल तथा सातताल झीलों का पानी के जल स्रोत पर इसका कुप्रभाव पड़ेगा। इसका वर्तमान में कुआताल जल स्रोत के सूखने तथा इलाके के सदाबहार जल स्रोत के गर्मी के मौसम में लगभग सूख जाने से स्पश्ट है। लंबे समयावधि में इन अमूल्य मीटे पानी के संषाधनों पर षहरीकरण के नकरात्मक असर का कोई सुधार तभी संभव है जब जोंस स्टेट को हरित पट्टी घोशित की जाये तथा भीमताल झील क जल संग्रहण क्षेत्र में निर्माण कार्य पर पाबंदी लगे जैसे कि उत्तराखण्ड मं नैनीताल तथा मसूरी में किया गया है

\section{INTRODUCTION}

Jones Estate ("June State" on Revenue Department records) is a forested microwatershed in Nainital district, Uttarakhand $\left(29^{0} 21^{\prime} 17^{\prime \prime} \mathrm{N} \&\right.$ 79'32'34.27'E), separating the Bhimtal and Sattal lake systems (Image 1). In the Himalaya, it is a unique geographical feature, being the only forested watershed separating two lake systems comprising a total of eight perennial and seasonal lakes. Bhimtal (tal = lake in Hindi) lies at the southeastern end while the Sattal lies along the northern half of the western face of the Estate. Comprising roughly 4.8 sq.km (1200 acres) of private forest in 1951, the forest area of Jones Estate has been reduced by roughly $30 \%$ due to cultivation and habitation over the years.

The lowest point is $1200 \mathrm{~m}$ at the conjoined Ram and Sita lakes of the Sattal (seven lakes) system, while the highest point is Thala at $1731 \mathrm{~m}$. The range runs northwest to southeast for a distance of roughly $3 \mathrm{~km}$ from Dhupchaura pass on the northwest to Tallital market and Bohrakun Village on its southeastern and southern faces respectively (Image 1). To the east lies the Bhimtal lake system comprising 


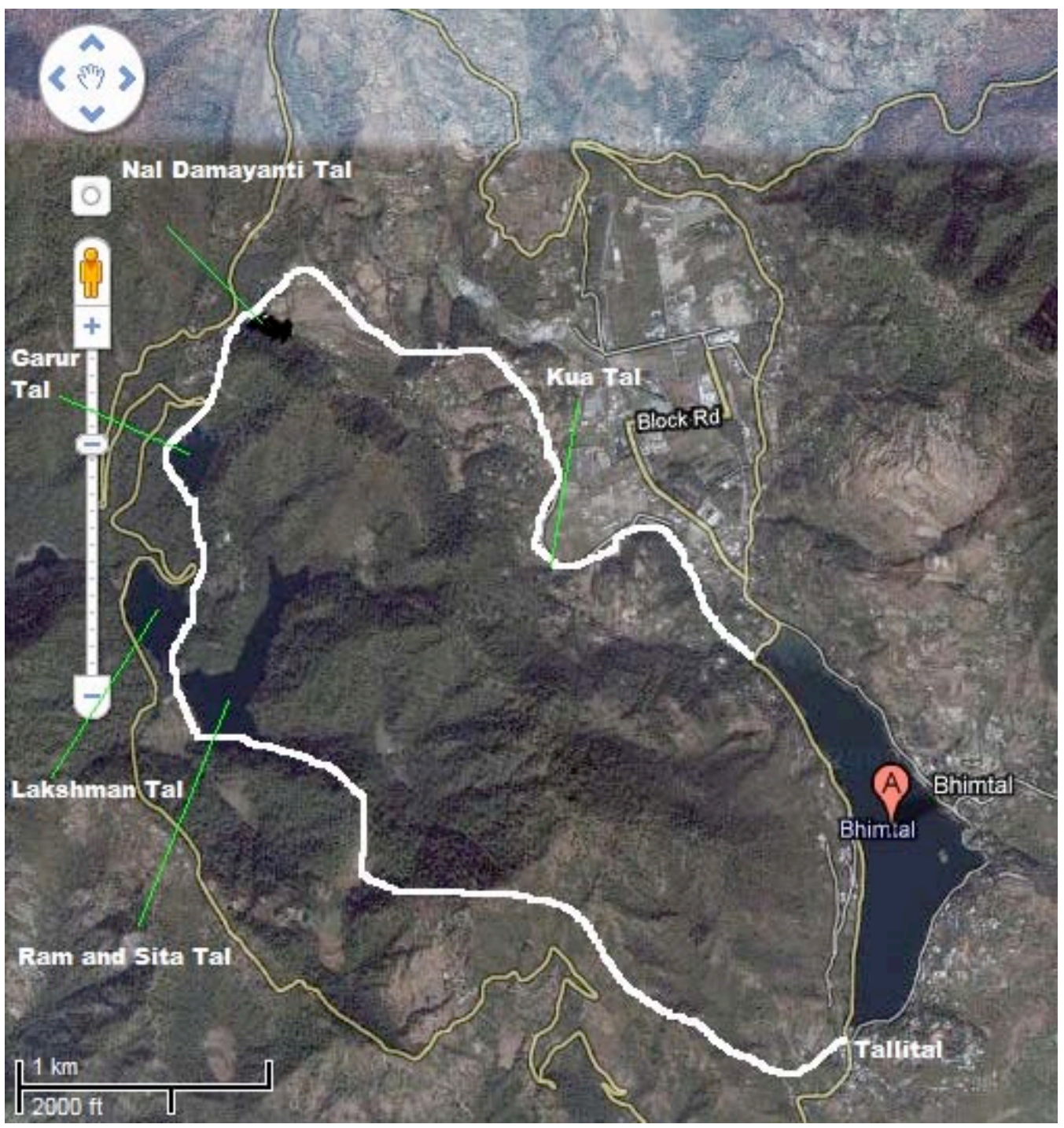

Image 1. Google map showing Jones Estate watershed outlined in white and adjoining lake systems. Source: Googlemaps

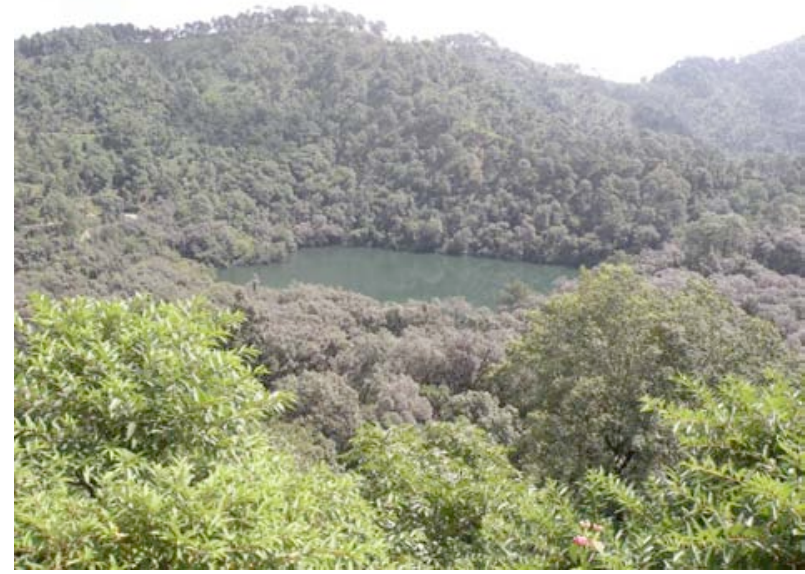

Image 2. Garurtal with the western face of Jones Estate in the background. of three lakes - Nal-Damayantital, Kuatal and Bhimtal. To the west lies the Sattal lake system comprising of Pannatal (=Garurtal) (Image 2); Ramtal and Sitatal, Lakshmantal, Sukhatal, Sariyatal and Lokhamtal.

Jones Estate lies in the outermost range of the Himalayan foothills and receives heavy rainfall. Although Osmaston (1927) gives a range of 2000$3000 \mathrm{~mm}$ of rainfall for this area, actual precipitation is rather less nowadays, averaging $1443 \mathrm{~mm}$ for the fiveyear period from 2005 to 2009 (Anonymous 2010).

The forest consists of three plant associations, namely sub-tropical broadleaf with Himalayan Oak Quercus leucotrichophora as a nodal species; Chir Pine Pinus roxburghii forest and elements of miscellaneous deciduous forest. In addition, there is 
a patch of naturalized Himalayan Cypress Cupressus torulosa several acres in extent.

\section{HISTORY}

TheBhimtal Valley has been inhabited and cultivated for over a millennium and Atkinson (1882) noted that it was one of the largest single sheets of cultivation in the Kumaon Himalaya.

Jones Estate watershed and the Sattal Valley were not inhabited during pastcenturies, although some small patches of cultivation were attempted by share-croppers and itinerant families until 1952. The major part of the Estate has always been forested. It came into existence in 1867 as a fee-simple estate, with the main aim of developing it for the production of green tea for the Tibetan market. Since then, it has remained in private hands.

On 17 January 2001 the then Minister for Environment and Forests, Mr. Kandari, stated in the Uttarakhand State Assembly that all concerned government departments, including the Public Works Department, Forest Department, Pollution Control Department, etc. in their reports on the possibility of construction of buildings on the Jones Estate watershed, had stressed that any such move would result in disastrous consequences for Bhimtal and for the water storage capability of the Sattal lakes (Special Correspondent 2001).

In 1954, a Forest Working Plan was passed for Jones Estate by the Forest Department. In the Land Record Settlement known as the Bandobast in 1957, land use of the greater part of Jones Estate was recorded as "forest". Under the provisions of the Forest Conservation Act 1980, no land use change is permitted on such land without the permission of the Central Government.

Despite this, numerous houses and resorts are being constructed and there is no doubt that the eventual urbanization of the Estate is well under way, to the detriment of the Bhimtal and Sattal lake systems and the wildlife inhabiting the Estate at present. Therefore, the present paper documents the butterflies recorded on the Estate (Table 1) so as to get a better idea of what is being lost and the eventual consequences of urbanization of the Jones Estate watershed.
The present paper also documents the butterflies and vertebrates afforded protection under the Schedules of the Indian Wildlife (Protection) Act 1972 (Anonymous 2006) that have been recorded on the Estate (Table 2). In total, these constitute 49 species, 11 on Schedule 1 and 39 on Schedule 2 (Hypolimnas misippus Linnaeus figures on both Schedules and is counted only once).

\section{MATERIAL AND METHODS}

The butterflies of the Estate have been studied since 1951. Some original specimens still exist, but the major resource from this era is in the form of notes maintained by my father, the late Fred Smetacek Sr. In the course of studying local butterflies, two butterfly subspecies new to science were discovered on the Estate, namely Neptis miah varshneyi Smetacek and Neptis clinia praedicta Smetacek (both Nymphalidae) (Smetacek 2002; 2011b). Besides, several butterflies previously unrecorded from the Western Himalaya have been reported (Smetacek 2010). Moths have been studied since 1972. Several species new to science have been described from the Estate (Smetacek 2002; 2005; 2010a). Besides, the population of hawkmoths (Lepidoptera: Sphingidae) on the Estate provided material for pioneering work in using members of an insect community as bio-indicators to predict and track climate change (Smetacek 1994; 2004).

The sightings of mammals and birds included in Annexure 2 were compiled mostly during the 1980s, when much time was spent patrolling the forest. They are all based on actual sightings by the author. The most recent sighting of a mammal protected under the provisions of the Wildlife (Protection) Act 1972 and listed as Near Threatened on the IUCN Red Data List is of a Himalayan Serow (Capricornis sumatraensis thar) which was sighted and photographed outside the Butterfly Research Centre, Jones Estate at $10 \mathrm{am}$ on 07 November 2011.

The use of a lepidopteran community as an indicator of forest health and consequently the health of the ecosystem including sub-surface water resources has been explored on the Estate by the author for the past 30 years (Smetacek 1993-2010). The format of Image 1, which enables a great deal of information to be presented concisely, has been taken with kind 
Table 1. Butterflies recorded in Jones Estate, Bhimtal, Between 1951 and 2011.

1 - Name

2 - Geographic distribution of the species.

3 - General distribution G - garden species, found everywhere; NA - widespread but only in undisturbed areas; LO - localized in colonies; RM - not resident, but regular migrants; SM - sporadic,rare migrants;

4 - Usual Habitat: U - universal across all habitats; SE - subtropical evergreen forest above 1200m; TD - tropical deciduous forest between 400 and 1400 m; SR - Shorea robusta forest below 1000m; S - scrubland; G - grassland.

5 - Relative abundance, sightings per day during seasonal optimum: $\mathrm{V}$ - very rare, none or one: R - rare (2-4); O - occasional (5-9); A - abundant (10-49); C - common (>50).

6 - Index of relative movement of an average individual during adult lifespan. The values are existential estimates and subject to error. 0 - moves less than 100m; 1 - moves 100-1000 m; 2 - moves 1-5 km; 3 - strongly migratory/dispersive.

7 - Voltinism, Number of complete life cycles per year. S - Univoltine, single generation; B - Bivoltine, two discrete generations; M - Multivoltine, multiple, usually overlapping generations. Months during which adults have been recorded are in parentheses eg (iii-vii).

8 - Stage of life cycle that diapauses: O - none; E - egg; L - larva; P pupa; A - adult.

9 - Span of larval food plant (FP) preferences: M - Monophagous, feeds on plant species within one genus; O - Oligophagous, feeds on plant genera within one family; P - Polyphagous, feeds on plants in two or more families.

10 - Larval food plant families; in cases where the spectrum of families is very wide, only two of the most important families in the area are included. For others, reference may be made to Robinson et al. (2001).

Throughout the paper, the symbol ? indicates the lack of dependable data.

\begin{tabular}{|c|c|c|c|c|c|c|c|c|c|}
\hline 1 & 2 & 3 & 4 & 5 & 6 & 7 & 8 & 9 & 10 \\
\hline \multicolumn{10}{|l|}{ PAPILIONIDAE } \\
\hline $\begin{array}{l}\text { 1. Troides aeacus } \\
\text { Felder \& Felder }\end{array}$ & $\begin{array}{l}\text { Himalaya from } \\
\text { Garhwal east to } \\
\text { China and Taiwan }\end{array}$ & SM & SE & $\mathrm{R}$ & 1 or 2 & B: v-vii. & ?P & 0 & Aristolochiaceae \\
\hline $\begin{array}{l}\text { 2. Atrophaneura } \\
\text { aidoneus Doubleday }\end{array}$ & $\begin{array}{l}\text { Himalaya from } \\
\text { Garhwal east to } \\
\text { Vietnam }\end{array}$ & LO & SE & $\mathrm{R}$ & 1 or 2 & $\begin{array}{l}\text { B: iv-v; } \\
\text { vii-viii }\end{array}$ & ?P & 0 & Aristolochiaceae \\
\hline $\begin{array}{l}\text { 03. Byasa dasarada } \\
\text { ravana Moore }\end{array}$ & $\begin{array}{l}\text { Himalaya from } \\
\text { Afghanistan to Indo- } \\
\text { China }\end{array}$ & ?RM/ LO & SE & 0 & 1 or 2 & $\begin{array}{l}\text { B:iv-vi; } \\
\text { vii-ix. }\end{array}$ & ?P & 0 & Aristolochiaceae \\
\hline $\begin{array}{l}\text { 04. Byasa polyeuctes } \\
\text { letincius Fruhstorfer }\end{array}$ & $\begin{array}{l}\text { Himalaya from } \\
\text { Afghanistan to } \\
\text { Vietnam }\end{array}$ & ?RM/ LO & SE & $\mathrm{R}$ & 1 or 2 & $\begin{array}{l}\text { B: iv-vi; } \\
\text { viii-ix }\end{array}$ & ?P & 0 & Aristolochiaceae \\
\hline $\begin{array}{l}\text { 05. Byasa latreillei } \\
\text { Donovan }\end{array}$ & $\begin{array}{l}\text { Afghanistan to China } \\
\text { and Vietnam. }\end{array}$ & SM & SE & V & ? & ?B: v; viii. & ?P & ? & Aristolochiaceae \\
\hline $\begin{array}{l}\text { 06. Pachliopta } \\
\text { aristolochiae } \\
\text { aristolochiae } \\
\text { Fabricius }\end{array}$ & $\begin{array}{l}\text { Afghanistan through } \\
\text { India to Japan and } \\
\text { Indonesia. }\end{array}$ & ?RM/NA & SR, S & $\mathrm{O}$ & $2 / 3$ & $\begin{array}{l}\text { M: i; iii-v; } \\
\text { x-xii. }\end{array}$ & $?$ & $P$ & $\begin{array}{l}\text { Aristolochiaceae; } \\
\text { Dioscoreaceae. }\end{array}$ \\
\hline $\begin{array}{l}\text { 07. Papilio agestor } \\
\text { govindra Moore }\end{array}$ & $\begin{array}{l}\text { West Himalaya to } \\
\text { Indo-China. }\end{array}$ & NA & SE & $\mathrm{R}$ & 0 to 2 & S: iii-v. & $P$ & 0 & Lauraceae \\
\hline $\begin{array}{l}\text { 08. Papilio clytia clytia } \\
\text { Linnaeus }\end{array}$ & India to Malaysia. & NA & $\begin{array}{l}\text { TD, } \\
\text { SR }\end{array}$ & 0 & 0 to 2 & $\begin{array}{l}\text { M: iii- viii; } \\
\quad \text { x. }\end{array}$ & ?P & $\mathrm{P}$ & $\begin{array}{l}\text { Lauraceae, } \\
\text { Sapotaceae }\end{array}$ \\
\hline $\begin{array}{l}\text { 09. Papilio protenor } \\
\text { protenor Cramer }\end{array}$ & Pakistan to Japan & NA & SE, S & $\mathrm{O}$ & 1 to 2 & M: ii-x. & $\mathrm{P}$ & $\mathrm{P}$ & $\begin{array}{l}\text { Lauraceae; } \\
\text { Rutaceae; } \\
\text { Polygalaceae. }\end{array}$ \\
\hline $\begin{array}{l}\text { 10. Papilio bianor } \\
\text { polyctor Boisduval }\end{array}$ & $\begin{array}{l}\text { Pakistan to Indo- } \\
\text { China. }\end{array}$ & NA & SE, S & 0 & 1 to 2 & $\begin{array}{l}\text { M: ii-vii; } \\
\text { viii-x. }\end{array}$ & $\mathrm{P}$ & 0 & Rutaceae \\
\hline $\begin{array}{l}\text { 11. Papilio paris } \\
\text { Linnaeus }\end{array}$ & $\begin{array}{l}\text { India to China and } \\
\text { S.E. Asia }\end{array}$ & NA & SE, S & $\mathrm{R}$ & 2 & $\begin{array}{l}\text { M: iii-v; } \\
\text { vii; x. }\end{array}$ & $? \mathrm{P}$ & 0 & Rutaceae \\
\hline $\begin{array}{l}\text { 12. Papilio polytes } \\
\text { romulus Cramer }\end{array}$ & $\begin{array}{l}\text { Pakistan throughout } \\
\text { India to Indonesia.. }\end{array}$ & $\mathrm{G}, \mathrm{NA}$ & $u$ & $\mathrm{~A} / \mathrm{C}$ & 2 & M: iii-x. & $\mathrm{P}$ & 0 & Rutaceae \\
\hline $\begin{array}{l}\text { 13. Papilio demoleus } \\
\text { Linnaeus }\end{array}$ & $\begin{array}{l}\text { Iran to China and } \\
\text { Australia. }\end{array}$ & $\mathrm{G}, \mathrm{NA}$ & u & O & 2,3 & M: iii-x. & ?P & 0 & Rutaceae \\
\hline $\begin{array}{l}\text { 14. Papilio machaon } \\
\text { Linnaeus }\end{array}$ & Palaearctic Region & G, LO & $\mathrm{S}, \mathrm{G}$ & 0 & $0-2$ & M: ii-vii. & $P$ & 0 & Umbelliferae \\
\hline $\begin{array}{l}\text { 15. Graphium(Pazala) } \\
\text { eurous cashmirensis } \\
\text { Rothschild }\end{array}$ & $\begin{array}{l}\text { West Himalaya to } \\
\text { Indo-China. }\end{array}$ & SM & SE & V & 1 to 2 & S: iv-v. & $\mathrm{P}$ & $\mathrm{O}$ & Lauraceae \\
\hline
\end{tabular}




\begin{tabular}{|c|c|c|c|c|c|c|c|c|c|}
\hline 1 & 2 & 3 & 4 & 5 & 6 & 7 & 8 & 9 & 10 \\
\hline $\begin{array}{l}\text { 16. Graphium } \\
\text { (Pathysa) nomius } \\
\text { Esper }\end{array}$ & India to Thailand. & SM & $\begin{array}{l}\text { TD } \\
\text { SR }\end{array}$ & V & 1,2 & B: iv-v; vii. & $?$ & $\mathrm{O}$ & Annonaceae \\
\hline $\begin{array}{l}\text { 17. Graphium } \\
\text { (Graphium) cloanthus } \\
\text { Westwood }\end{array}$ & Himalaya to China & NA & SE & $\mathrm{O}$ & 1,2 & $\begin{array}{l}\text { M: iii-vi; } \\
\text { viii-x. }\end{array}$ & $\mathrm{P}$ & $\mathrm{O}$ & Lauraceae \\
\hline $\begin{array}{l}\text { 18. Graphium } \\
\text { (Graphium) sarpedon } \\
\text { Linnaeus }\end{array}$ & $\begin{array}{l}\text { India to Japan and } \\
\text { Australia. }\end{array}$ & NA & SE & $\mathrm{O}$ & 1,2 & M: ii-x. & $\mathrm{P}$ & $\mathrm{P}$ & $\begin{array}{l}\text { Lauraceae; } \\
\text { Annonaceae }\end{array}$ \\
\hline $\begin{array}{l}\text { 19. Graphium } \\
\text { (Graphium) } \\
\text { agamemnon } \\
\text { Linnaeus }\end{array}$ & $\begin{array}{l}\text { India to China and } \\
\text { Australia. }\end{array}$ & RM & SR & V & 3 & $\begin{array}{l}\text { B or M: iii- } \\
\text { iv; viii. }\end{array}$ & $?$ & $\mathrm{P}$ & $\begin{array}{l}\text { Annonaceae; } \\
\text { Magnoliaceae; etc }\end{array}$ \\
\hline \multicolumn{10}{|l|}{ PIERIDAE } \\
\hline $\begin{array}{l}\text { 20. Leptosia nina } \\
\text { Fabricius }\end{array}$ & $\begin{array}{l}\text { India to the } \\
\text { Philippines. }\end{array}$ & LO & $\begin{array}{l}\text { TD, } \\
\text { SR }\end{array}$ & V & $\mathrm{O}$ & $\begin{array}{l}\text { M: ii-v; } \\
\text { ix-xii. }\end{array}$ & $?$ & $\mathrm{O}$ & Capparaceae \\
\hline $\begin{array}{l}\text { 21. Pontia daplidice } \\
\text { moorei Röber }\end{array}$ & Palaearctic. & G, NA & S, G & $\mathrm{O}$ & 2,3 & $\begin{array}{l}\text { M: iii-vii; } \\
\text { ix-x; xii. }\end{array}$ & ?P & $\mathrm{O}$ & Cruciferae \\
\hline $\begin{array}{l}\text { 22. Pieris canidia } \\
\text { indica Evans }\end{array}$ & India to China. & G, NA & $U$ & A & 2 & $\begin{array}{l}\text { M: i-v; vii; } \\
\text { ix-xii. }\end{array}$ & ?P & $\mathrm{O}$ & Cruciferae \\
\hline $\begin{array}{l}\text { 23. Pieris brassicae } \\
\text { nipalensis Gray }\end{array}$ & Palaearctic. & G, NA & $U$ & C & 3 & M: iii-xi. & ?P & $\mathrm{P}$ & $\begin{array}{l}\text { Cruciferae; } \\
\text { Resedaceae; etc. }\end{array}$ \\
\hline $\begin{array}{l}\text { 24. Aporia agathon } \\
\text { Gray }\end{array}$ & Himalaya to China & NA & SE & A & 0 to 2 & S: iv-vi. & $?$ & $\mathrm{O}$ & Berberidaceae \\
\hline $\begin{array}{l}\text { 25. Delias eucharis } \\
\text { Drury }\end{array}$ & Throughout India & RM & $\begin{array}{l}\text { TD, } \\
\text { SR }\end{array}$ & $\mathrm{O}$ & $2-3$ & $\begin{array}{l}\text { M: i-v; } \\
\text { x-xii, }\end{array}$ & $?$ & $\mathrm{P}$ & $\begin{array}{l}\text { Loranthaceae; } \\
\text { Malvaceae; etc. }\end{array}$ \\
\hline $\begin{array}{l}\text { 26. Delias belladonna } \\
\text { horsfieldii Gray }\end{array}$ & $\begin{array}{l}\text { West Himalaya to } \\
\text { China. }\end{array}$ & LO & SE & $\mathrm{O}$ & 1 to 2 & $\begin{array}{l}\text { M: ii-v; } \\
\text { viii-x. }\end{array}$ & $?$ & $\mathrm{O}$ & Loranthaceae \\
\hline $\begin{array}{l}\text { 27. Delias acalis } \\
\text { Godart }\end{array}$ & $\begin{array}{l}\text { Himalaya to Indo- } \\
\text { China }\end{array}$ & SM & TD & V & 2 to 3 & B: iii-iv; ix. & $?$ & $?$ & $?$ \\
\hline $\begin{array}{l}\text { 28. Anaphaeis aurota } \\
\text { Fabricius }\end{array}$ & $\begin{array}{l}\text { Throughout India to } \\
\text { S.E. Asia }\end{array}$ & RM & SR, S & A & 3 & $\begin{array}{l}\text { M: iv-v; viii- } \\
\quad \text {;;xii. }\end{array}$ & $?$ & $\mathrm{P}$ & $\begin{array}{l}\text { Capparaceae; } \\
\text { Oleaceae }\end{array}$ \\
\hline $\begin{array}{l}\text { 29. Cepora nerissa } \\
\text { phryne Fabricius }\end{array}$ & $\begin{array}{l}\text { Throughout India to } \\
\text { S.E. Asia. }\end{array}$ & RM & SR, S & A & 3 & $\begin{array}{l}\text { M: iii-iv; } \\
\text { vi-xii. }\end{array}$ & $?$ & $\mathrm{O}$ & Capparaceae \\
\hline $\begin{array}{l}\text { 30. Appias lalage } \\
\text { lalage Doubleday }\end{array}$ & $\begin{array}{l}\text { Himalaya to Indo- } \\
\text { China. }\end{array}$ & RM & $?$ & $\mathrm{O}$ & 3 & $\begin{array}{l}\text { M: ii-v; viii; } \\
\quad \text { xii. }\end{array}$ & $?$ & $?$ & Leguminosae \\
\hline $\begin{array}{l}\text { 31. Catopsilia } \\
\text { pomona Fabricius }\end{array}$ & $\begin{array}{l}\text { Indo-Australian } \\
\text { Region. }\end{array}$ & RM & $U$ & C & 3 & M: i-xii. & $?$ & $\mathrm{O}$ & Leguminosae \\
\hline $\begin{array}{l}\text { 32. Catopsilia } \\
\text { pyranthe minna } \\
\text { Herbst }\end{array}$ & $\begin{array}{l}\text { Throughout India to } \\
\text { S.E. Asia }\end{array}$ & RM & $U$ & C & 3 & M: ii-x. & $?$ & $\mathrm{O}$ & Leguminosae \\
\hline $\begin{array}{l}\text { 33. Gonepteryx } \\
\text { rhamni nepalensis } \\
\text { Doubleday }\end{array}$ & Palaearctic Region. & NA & SE, S & $\mathrm{O}$ & 1 to 2 & M: i-xii. & A & $? \mathrm{P}$ & $\begin{array}{l}\text { Rhamnaceae; } \\
\text { Ericaceae }\end{array}$ \\
\hline $\begin{array}{l}\text { 34. Eurema brigitta } \\
\text { rubella Wallace }\end{array}$ & $\begin{array}{l}\text { Africa through India } \\
\text { to Malaysia. }\end{array}$ & G, NA & $U$ & A & 0 to 3 & M: i-xii. & $?$ & $\mathrm{P}$ & $\begin{array}{l}\text { Leguminosae; } \\
\text { Guttiferae; etc. }\end{array}$ \\
\hline $\begin{array}{l}\text { 35. Eurema laeta } \\
\text { Boisduval }\end{array}$ & $\begin{array}{l}\text { India to Japan and } \\
\text { Australia. }\end{array}$ & G, NA & $U$ & A & 0 to ?2 & $\begin{array}{l}\text { M: i; iii-iv; } \\
\text { vi-xii. }\end{array}$ & $?$ & $\mathrm{O}$ & Leguminosae \\
\hline $\begin{array}{l}\text { 36. Eurema hecabe } \\
\text { fimbriata Wallace }\end{array}$ & $\begin{array}{l}\text { African and Indo- } \\
\text { Australian Regions }\end{array}$ & G, NA & $U$ & C & 0 to ?2 & M: i-xii. & $?$ & $\mathrm{P}$ & $\begin{array}{l}\text { Leguminosae; } \\
\text { Euphorbiaceae; } \\
\text { etc. }\end{array}$ \\
\hline $\begin{array}{l}\text { 37. Colias fieldii } \\
\text { Menétries }\end{array}$ & $\begin{array}{l}\text { Eastern Palaearctic } \\
\text { Region }\end{array}$ & G, NA & SE, G & A & 3 & M: i-xii. & $?$ & $?$ & $?$ \\
\hline $\begin{array}{l}\text { 38. Colias erate } \\
\text { Esper }\end{array}$ & Palaearctic Region & NA & SE, G & $\mathrm{O}$ & 3 & M: i-xi. & $?$ & $\mathrm{O}$ & Leguminosae \\
\hline $\begin{array}{l}\text { 39. Ixias pyrene } \\
\text { Linnaeus }\end{array}$ & India to China. & SM & S & V & 3 & $\begin{array}{l}\text { B: ix-x; } \\
\text { xii-i. }\end{array}$ & $?$ & $\mathrm{O}$ & Capparaceae \\
\hline $\begin{array}{l}\text { 40. Ixias marianne } \\
\text { Cramer }\end{array}$ & India & SM & $S$ & V & 3 & B: i-iv; xii. & $?$ & $\mathrm{O}$ & Capparaceae \\
\hline $\begin{array}{l}\text { 41. Pareronia valeria } \\
\text { Cramer }\end{array}$ & $\begin{array}{l}\text { India to the } \\
\text { Philippines. }\end{array}$ & RM & $\begin{array}{l}\text { TD, } \\
\text { SR }\end{array}$ & $\mathrm{R}$ & 3 & $\begin{array}{l}\text { M: iii-v; } \\
\text { x-xii. }\end{array}$ & $?$ & $\mathrm{O}$ & Capparaceae \\
\hline \multicolumn{10}{|l|}{ NYMPHALIDAE } \\
\hline $\begin{array}{l}\text { 42. Parantica aglea } \\
\text { melanoides Moore }\end{array}$ & $\begin{array}{l}\text { Himalaya to S.E. } \\
\text { Asia. }\end{array}$ & G, NA & $\begin{array}{l}\text { SE, } \\
\text { TD }\end{array}$ & $\mathrm{O}$ & 0 to 2 & $\begin{array}{l}\text { M: ii-v; } \\
\text { vii-x; xii. }\end{array}$ & $?$ & $\mathrm{O}$ & Asclepiadaceae \\
\hline
\end{tabular}




\begin{tabular}{|c|c|c|c|c|c|c|c|c|c|}
\hline 1 & 2 & 3 & 4 & 5 & 6 & 7 & 8 & 9 & 10 \\
\hline $\begin{array}{l}\text { 43. Parantica sita } \\
\text { Kollar }\end{array}$ & $\begin{array}{l}\text { Himalaya to Japan } \\
\text { and Malaysia. }\end{array}$ & NA & SE & $\mathrm{R}$ & 1 to 2 & $\begin{array}{l}\text { B: iii-v; } \\
\text { ix-xi. }\end{array}$ & $?$ & $\mathrm{O}$ & Asclepiadaceae. \\
\hline $\begin{array}{l}\text { 44. Tirumala limniace } \\
\text { mutina Fruhstorfer }\end{array}$ & Oriental Region & NA & U & $\mathrm{O}$ & 2 to 3 & M: iii-x. & $?$ & $? 0$ & Asclepiadaceae \\
\hline $\begin{array}{l}\text { 45. Tirumala } \\
\text { septentrionis } \\
\text { septentrionis Butler }\end{array}$ & India to China & NA & $\begin{array}{l}\text { NA/ } \\
\text { ?RM }\end{array}$ & $\mathrm{O}$ & 3 & B: iii-v;ix-x. & $?$ & $P$ & $\begin{array}{l}\text { Apocynaceae; } \\
\text { Asclepiadaceae; } \\
\text { etc. }\end{array}$ \\
\hline $\begin{array}{l}\text { 46. Danaus } \\
\text { chrysippus Linnaeus }\end{array}$ & $\begin{array}{l}\text { African, Oriental and } \\
\text { Australian Regions }\end{array}$ & $\mathrm{G}, \mathrm{NA}$ & $U$ & A & 1 to 2 & M: i-xii. & $\mathrm{P}$ & $\mathrm{P}$ & $\begin{array}{l}\text { Asclepiadaceae; } \\
\text { Moraceae; etc. }\end{array}$ \\
\hline $\begin{array}{l}\text { 47. Danaus genutia } \\
\text { Cramer }\end{array}$ & Oriental Region & $\mathrm{G}, \mathrm{NA}$ & $U$ & $A$ & 1 to 2 & M: i-xii. & $\mathrm{P}$ & $\mathrm{O}$ & Asclepiadaceae \\
\hline $\begin{array}{l}\text { 48. Euploea mulciber } \\
\text { mulciber Cramer }\end{array}$ & $\begin{array}{l}\text { Himalaya to S.E. } \\
\text { Asia }\end{array}$ & NA & $\begin{array}{l}\text { SE, } \\
\text { TD, }\end{array}$ & $\mathrm{O}$ & 0 to 2 & $\begin{array}{l}\text { M: iii-vii; } \\
\text { ix-x. }\end{array}$ & $? P$ & $P$ & $\begin{array}{l}\text { Asclepidiaceae; } \\
\text { Moraceae; etc. }\end{array}$ \\
\hline $\begin{array}{l}\text { 49. Euploea core } \\
\text { core Cramer }\end{array}$ & $\begin{array}{l}\text { Indo-Australian } \\
\text { Region }\end{array}$ & NA & $\begin{array}{l}\text { SE, } \\
\text { TD, } \\
\text { SR }\end{array}$ & C & 0 to 3 & $\begin{array}{l}\text { M: ii-vii; } \\
\text { ix-x. }\end{array}$ & $\mathrm{P}$ & $P$ & $\begin{array}{l}\text { Asclepidiaceae; } \\
\text { Moraceae; etc. }\end{array}$ \\
\hline $\begin{array}{l}\text { 50. Euploea sylvester } \\
\text { Fabricius }\end{array}$ & India to S.E. Asia & SM & $?$ & V & 0 to 3 & ?S: iv. & $?$ & $P$ & $\begin{array}{l}\text { Ascelpidiaceae; } \\
\text { Moraceae; etc. }\end{array}$ \\
\hline $\begin{array}{l}\text { 51. Mycalesis } \\
\text { perseus Fabricius }\end{array}$ & $\begin{array}{l}\text { Indo-Australian } \\
\text { Region }\end{array}$ & NA & $\begin{array}{l}\text { TD, } \\
\text { SR }\end{array}$ & A & 0 to 1 & M: iii-v; x. & $?$ & $\mathrm{O}$ & Graminae \\
\hline $\begin{array}{l}\text { 52. Mycalesis mineus } \\
\text { Linnaeus }\end{array}$ & India to China & NA & $\begin{array}{l}\text { TD, } \\
\text { SR }\end{array}$ & $\mathrm{O}$ & 0 to 1 & M: iii-v; x. & $?$ & $\mathrm{O}$ & Graminae \\
\hline $\begin{array}{l}\text { 53. Mycalesis visala } \\
\text { visala Moore }\end{array}$ & India to Malaysia & NA & $\begin{array}{l}\text { TD, } \\
\text { SR }\end{array}$ & $\mathrm{O}$ & 0 to 1 & B: iv-vi; x. & $?$ & $\mathrm{O}$ & Graminae \\
\hline $\begin{array}{l}\text { 54. Mycalesis heri } \\
\text { Moore }\end{array}$ & Himalaya & NA & TD & $\mathrm{O}$ & 0 to 1 & $\begin{array}{l}\text { B: iv-vi; } \\
\text { viii-x. }\end{array}$ & $?$ & $?$ & $?$ \\
\hline $\begin{array}{l}\text { 55. Lethe rohria dyrta } \\
\text { Felder \& Felder }\end{array}$ & India to China & NA & $\begin{array}{l}\text { SE, } \\
\text { TD }\end{array}$ & $\mathrm{C}$ & 0 to 2 & M: iii-ix. & $?$ & $\mathrm{O}$ & Graminae \\
\hline $\begin{array}{l}\text { 56. Lethe confusa } \\
\text { confusa Aurivillius }\end{array}$ & $\begin{array}{l}\text { Himalaya to China } \\
\text { and Malaysia. }\end{array}$ & NA & $\begin{array}{l}\text { SE, } \\
\text { TD }\end{array}$ & $\mathrm{O}$ & 0 to 2 & M: iii-x. & $?$ & $\mathrm{O}$ & Graminae \\
\hline $\begin{array}{l}\text { 57. Lethe kansa } \\
\text { Moore }\end{array}$ & Himalaya & NA & SE & $\mathrm{R}$ & 0 to 2 & B: iii-v; $x$ & $?$ & $?$ & $?$ \\
\hline $\begin{array}{l}\text { 58. Lethe verma } \\
\text { verma Kollar }\end{array}$ & $\begin{array}{l}\text { Himalaya to China } \\
\text { and Malaysia }\end{array}$ & NA & SE & $\mathrm{R}$ & 0 to 1 & $\begin{array}{l}\text { B: v-vi; } \\
\text { viii-ix. }\end{array}$ & $?$ & $? 0$ & Graminae \\
\hline $\begin{array}{l}\text { 59. Lasiommata } \\
\text { schakra Kollar }\end{array}$ & Himalaya & NA, LO & S, G & $\mathrm{R}$ & 0 to 1 & M: iii-ix. & $?$ & $?$ & $?$ \\
\hline $\begin{array}{l}\text { 60. Orinoma damaris } \\
\text { Gray }\end{array}$ & Himalaya & NA & SE & $\mathrm{R}$ & 0 to 1 & $\begin{array}{l}\text { B: iv-vi; } \\
\text { ix-x. }\end{array}$ & $?$ & $?$ & $?$ \\
\hline $\begin{array}{l}\text { 61. Aulocera swaha } \\
\text { swaha Kollar }\end{array}$ & Himalaya & SM & S, G & V & 0 to 2 & S: vii-x. & $?$ & $?$ & ?Graminae \\
\hline $\begin{array}{l}\text { 62. Aulocera } \\
\text { saraswati Kollar }\end{array}$ & Himalaya & SM & S, G & V & 0 to 2 & S: viii-ix. & $?$ & $?$ & ?Graminae \\
\hline $\begin{array}{l}\text { 63. Callerebia hybrida } \\
\text { Butler }\end{array}$ & $\begin{array}{l}\text { West Himalayan } \\
\text { endemic }\end{array}$ & NA & S, G & A & 0 to 2 & $\begin{array}{l}\text { B: v-vi; } \\
\text { viii-x. }\end{array}$ & $?$ & $?$ & $?$ \\
\hline $\begin{array}{l}\text { 64. Callerebia annada } \\
\text { caeca Watkins }\end{array}$ & Himalaya to China & NA & $\begin{array}{l}\mathrm{SE} \\
\mathrm{S}, \mathrm{G}\end{array}$ & $\mathrm{O}$ & 0 to 2 & $\begin{array}{l}\text { B: iii-vi; } \\
\text { viii-x. }\end{array}$ & $?$ & $?$ & $?$ \\
\hline $\begin{array}{l}\text { 65. Ypthima nareda } \\
\text { nareda Kollar }\end{array}$ & Himalaya to China & NA & $\begin{array}{l}\mathrm{SE} \\
\mathrm{S}, \mathrm{G}\end{array}$ & $\mathrm{O}$ & 1 to 2 & M: iv-x. & $?$ & $\mathrm{O}$ & Graminae. \\
\hline $\begin{array}{l}\text { 66. Ypthima hubneri } \\
\text { hubneri Kirby }\end{array}$ & Himalaya & $\mathrm{G}, \mathrm{NA}$ & $\begin{array}{l}\text { TD, } \\
\mathrm{S}, \mathrm{G}\end{array}$ & $\mathrm{O}$ & 1 to 2 & $\begin{array}{l}\text { M: vi-viii; } \\
\text { xii-iii. }\end{array}$ & $?$ & $\mathrm{O}$ & Graminae. \\
\hline $\begin{array}{l}\text { 67. Ypthima lisandra } \\
\text { avanta Moore }\end{array}$ & India to Myanmar & NA. & $\begin{array}{l}\mathrm{SE} ; \\
\mathrm{S} ; \mathrm{G} .\end{array}$ & $\mathrm{O}$ & 1 to 2 & $\begin{array}{l}\text { M: xii-iii; } \\
\text { vi-viii. }\end{array}$ & $?$ & $? 0$ & Graminae \\
\hline $\begin{array}{l}\text { 68. Ypthima baldus } \\
\text { Fabricius }\end{array}$ & $\begin{array}{l}\text { India to Korea, } \\
\text { Japan and Malaysia }\end{array}$ & $\mathrm{G}, \mathrm{NA}$ & $U$ & $\mathrm{O}$ & 1 to 2 & M: vi-iii. & $?$ & $\mathrm{O}$ & Graminae. \\
\hline $\begin{array}{l}\text { 69. Ypthima asterope } \\
\text { mahratta Moore }\end{array}$ & India & NA. & $\begin{array}{l}\text { TD; } \\
\text { SR. }\end{array}$ & $C$ & 1 to 2 & B: viii-x. & $?$ & $\mathrm{O}$ & Graminae. \\
\hline $\begin{array}{l}\text { 70. Ypthima nikaea } \\
\text { Moore }\end{array}$ & Himalaya & NA & SE, G & A & 1 to 2 & M: v-x. & $?$ & $?$ & $?$ \\
\hline $\begin{array}{l}\text { 71. Melanitis leda } \\
\text { Linnaeus }\end{array}$ & $\begin{array}{l}\text { African, East } \\
\text { Palaearctic and } \\
\text { Indo-Australian } \\
\text { Regions }\end{array}$ & $\mathrm{G}, \mathrm{NA}$ & $U$ & $C$ & 0 to 2 & M: v-ix. & $?$ & $\mathrm{P}$ & $\begin{array}{l}\text { Graminae; } \\
\text { Palmae. }\end{array}$ \\
\hline
\end{tabular}




\begin{tabular}{|c|c|c|c|c|c|c|c|c|c|}
\hline 1 & 2 & 3 & 4 & 5 & 6 & 7 & 8 & 9 & 10 \\
\hline $\begin{array}{l}\text { 72. Melanitis phedima } \\
\text { galkissa Fruhstorfer }\end{array}$ & India to Malaysia. & NA & $\begin{array}{l}\text { SE, } \\
\text { TD, } \\
\text { SR }\end{array}$ & $\mathrm{R}$ & 0 to 2 & M: v-x. & $?$ & $\mathrm{O}$ & Graminae \\
\hline $\begin{array}{l}\text { 73. Melanitis zitenius } \\
\text { Herbst }\end{array}$ & Oriental Region & LO & TD & $\mathrm{R}$ & 0 to 2 & S: viii. & $?$ & $\mathrm{O}$ & Graminae. \\
\hline $\begin{array}{l}\text { 74. Elymnias } \\
\text { hypermnestra } \\
\text { undularis Drury }\end{array}$ & India to Malaysia. & LO. & SR. & $\mathrm{R}$. & 0 to 2 & M: iv-xi. & $?$ & $\mathrm{O}$ & Palmae \\
\hline $\begin{array}{l}\text { 75. Elymnias malelas } \\
\text { nilamba Fruhstorfer }\end{array}$ & $\begin{array}{l}\text { Himalaya to } \\
\text { Malaysia. }\end{array}$ & LO & TD & $\mathrm{R}$ & 0 to 2 & $\begin{array}{l}\text { M: iv-v; } \\
\text { viii-xi. }\end{array}$ & $?$ & ?M & Musaceae \\
\hline $\begin{array}{l}\text { 76. Polyura athamas } \\
\text { Drury }\end{array}$ & Oriental Region & NA & $\begin{array}{l}\text { SE, } \\
\text { TD }\end{array}$ & $\mathrm{O}$ & 0 to 2 & M: iv; vi-x. & $?$ & $\mathrm{P}$ & $\begin{array}{l}\text { Leguminosae; } \\
\text { Tiliaceae. }\end{array}$ \\
\hline $\begin{array}{l}\text { 77. Polyura agraria } \\
\text { Swinhoe }\end{array}$ & Oriental Region & NA & $\begin{array}{l}\text { TD, } \\
\text { SR }\end{array}$ & $\mathrm{R}$ & $?$ & B: iv; ix-x. & $?$ & $?$ & $?$ \\
\hline $\begin{array}{l}\text { 78. Polyura dolon } \\
\text { Westwood }\end{array}$ & $\begin{array}{l}\text { Himalayan to S.E. } \\
\text { Asia }\end{array}$ & SM & SE & V & 0 to 2 & B: v; ix. & $?$ & $?$ & $?$ \\
\hline $\begin{array}{l}\text { 79. Dilipa morgiana } \\
\text { Westwood }\end{array}$ & Himalaya & ?SM/LO & SE & V & $?$ & $\begin{array}{l}\text { M: iii; v; } \\
\text { viii. }\end{array}$ & $?$ & $?$ & $?$ \\
\hline $\begin{array}{l}\text { 80. Apatura } \\
\text { (Mimathyma) ambica } \\
\text { ambica Kollar }\end{array}$ & Himalaya & ?SM/LO & SE & V & $?$ & M: iv-x. & $?$ & $\mathrm{M}$ & Ulmaceae \\
\hline $\begin{array}{l}\text { 81. Sephisa dichroa } \\
\text { Kollar }\end{array}$ & $\begin{array}{l}\text { West Himalayan } \\
\text { endemic }\end{array}$ & LO & SE & A & 0 to 1 & M: v-x. & ?P & $\mathrm{M}$ & Fagaceae \\
\hline $\begin{array}{l}\text { 82. Euripus consimilis } \\
\text { Westwood }\end{array}$ & $\begin{array}{l}\text { Himalaya to S.E. } \\
\text { Asia, S. India }\end{array}$ & LO & $\begin{array}{l}\text { TD, } \\
\text { SR }\end{array}$ & $\mathrm{R}$ & 0 to 1 & $\begin{array}{l}\text { B: iii-v; } \\
\text { vii-ix. }\end{array}$ & $?$ & $\mathrm{O}$ & Ulmaceae \\
\hline $\begin{array}{l}\text { 83. Hestina persimilis } \\
\text { zella Butler }\end{array}$ & Himalaya & LO & SE & $\mathrm{R}$ & 1 to 2 & M: iii-x. & $?$ & $\mathrm{O}$ & Ulmaceae \\
\hline $\begin{array}{l}\text { 84. Hestinalis nama } \\
\text { Doubleday }\end{array}$ & $\begin{array}{l}\text { Himalaya to } \\
\text { Malaysia }\end{array}$ & NA & $\begin{array}{l}\text { SE, } \\
\text { TD }\end{array}$ & $\mathrm{R}$ & 1 to 2 & $\begin{array}{l}\text { M: ii-vi; } \\
\text { ix-x. }\end{array}$ & $?$ & $?$ & Urticaceae \\
\hline $\begin{array}{l}\text { 85. Dichorragia } \\
\text { nesimachus } \\
\text { Boisduval } \\
\end{array}$ & $\begin{array}{l}\text { Himalaya to Japan } \\
\text { and Philippines }\end{array}$ & ?SM/LO & SE & V & $? 1$ & B: iii-iv; viii. & $?$ & $\mathrm{O}$ & Meliosmaceae \\
\hline $\begin{array}{l}\text { 86. Stibochiona nicea } \\
\text { nicea Gray }\end{array}$ & $\begin{array}{l}\text { Himalaya to } \\
\text { Malaysia. }\end{array}$ & LO & $\begin{array}{l}\text { SE/ } \\
\text { TD }\end{array}$ & $\mathrm{R}$ & 1 to 2 & $\begin{array}{l}\text { M: iv-vii; } \\
\text { ix-x. }\end{array}$ & $?$ & $\mathrm{P}$ & $\begin{array}{l}\text { Moraceae; } \\
\text { Urticaceae. }\end{array}$ \\
\hline $\begin{array}{l}\text { 87. Tanaecia julii } \\
\text { appiades Menétries }\end{array}$ & $\begin{array}{l}\text { Himalaya to Indo- } \\
\text { China }\end{array}$ & SM & $\begin{array}{l}\mathrm{TD} / \\
\mathrm{SR}\end{array}$ & V & $?$ & $?: \mathrm{v}$ & $?$ & $? 0$ & Sapotaceae \\
\hline $\begin{array}{l}\text { 88. Euthalia aconthea } \\
\text { Hewitson }\end{array}$ & Oriental Region & G & $\begin{array}{l}\text { TD, } \\
\text { SR }\end{array}$ & $\mathrm{O}$ & 0 to 2 & $\begin{array}{l}\text { M: iii-iv; vi- } \\
\text { vii; x-xi. }\end{array}$ & ?P & $P$ & $\begin{array}{l}\text { Anacardiaceae; } \\
\text { Moraceae; etc. }\end{array}$ \\
\hline $\begin{array}{l}\text { 89. Euthalia Iubentina } \\
\text { indica Fruhstorfer }\end{array}$ & India to Malaysia. & NA & $\begin{array}{l}\text { TD, } \\
\text { SR }\end{array}$ & $\mathrm{R}$ & 0 to 2 & $\begin{array}{l}\text { M: iii; v; } \\
\text { viii, x-xi. }\end{array}$ & $?$ & $\mathrm{O}$ & Loranthaceae \\
\hline $\begin{array}{l}\text { 90. Euthalia patala } \\
\text { patala Kollar }\end{array}$ & $\begin{array}{l}\text { West Himalaya to } \\
\text { China }\end{array}$ & LO & SE & $\mathrm{O}$ & 0 to 2 & S: v-viii. & $\mathrm{P}$ & $\mathrm{O}$ & Fagaceae \\
\hline $\begin{array}{l}\text { 91. Symphaedra nais } \\
\text { Forst }\end{array}$ & Indian Subcontinent. & SM & SR & V & 0 to 2 & ?B: iv; ix. & $?$ & $P$ & $\begin{array}{l}\text { Dipteracarpaceae; } \\
\text { Ebenaceae. }\end{array}$ \\
\hline $\begin{array}{l}\text { 92. Auzakia danava } \\
\text { Moore }\end{array}$ & $\begin{array}{l}\text { Himalaya to } \\
\text { Myanmar }\end{array}$ & NA & SE & $\mathrm{R}$ & 0 to 2 & $\begin{array}{l}\text { M: iv-v; } \\
\text { viii; } x \text {. }\end{array}$ & $?$ & $?$ & $?$ \\
\hline $\begin{array}{l}\text { 93. Moduza procris } \\
\text { procris Cramer }\end{array}$ & $\begin{array}{l}\text { India to Philippines } \\
\text { and Indonesia. }\end{array}$ & SM & SR & V & 1 to 2 & ?M: iv; x-i. & $?$ & $P$ & $\begin{array}{l}\text { Rubiaceae; } \\
\text { Capparaceae. }\end{array}$ \\
\hline $\begin{array}{l}\text { 94. Athyma cama } \\
\text { Moore }\end{array}$ & Himalaya & NA & SE & $\mathrm{O}$ & 0 to 2 & M: iv-x. & $?$ & $?$ & Euphorbiaceae \\
\hline $\begin{array}{l}\text { 95. Athyma } \\
\text { selenophora } \\
\text { selenophora Kollar }\end{array}$ & India to China & NA & $\begin{array}{l}\text { TD, } \\
\text { SR }\end{array}$ & $\mathrm{R}$ & 0 to 2 & M: ii-vi; xi. & $?$ & $?$ & Rubiaceae \\
\hline $\begin{array}{l}\text { 96. Athyma opalina } \\
\text { opalina Kollar }\end{array}$ & Himalaya & NA & SE & $\mathrm{O}$ & 0 to 2 & M: iii-xii. & $\mathrm{P}$ & $\mathrm{O}$ & Berberidaceae \\
\hline $\begin{array}{l}\text { 97. Athyma perius } \\
\text { Linnaeus }\end{array}$ & $\begin{array}{l}\text { India to China and } \\
\text { Malaysia. }\end{array}$ & NA & TD & $\mathrm{O}$ & 0 to 2 & M: iv-xii. & $?$ & $\mathrm{O}$ & Euphorbiaceae \\
\hline $\begin{array}{l}\text { 98. Athyma asura } \\
\text { Moore }\end{array}$ & Himalaya. & SM & SE & V & $?$ & ?B: vi; viii. & $?$ & $?$ & Rubiaceae \\
\hline $\begin{array}{l}\text { 99. Phaedyma } \\
\text { columella ophiana } \\
\text { Moore }\end{array}$ & Himalaya. & SM & $\begin{array}{l}\text { TD, } \\
\text { SR }\end{array}$ & V & 0 to 2 & $\begin{array}{l}\text { M: iv; vi-vii; } \\
\quad \text { xii-i. }\end{array}$ & $?$ & $P$ & $\begin{array}{l}\text { Guttiferae; } \\
\text { Leguminosae; etc. }\end{array}$ \\
\hline $\begin{array}{l}\text { 100. Neptis nata } \\
\text { yerburii Butler }\end{array}$ & India to Borneo. & LO & $\begin{array}{l}\text { SE, } \\
\text { TD, } \\
\text { SR }\end{array}$ & $\mathrm{O}$ & 0 to 2 & $\begin{array}{l}\text { M: iii- vi; } \\
\text { ix-x. }\end{array}$ & $?$ & ?P & $\begin{array}{l}\text { ?Ulmaceae; } \\
\text { ?Combretaceae. }\end{array}$ \\
\hline
\end{tabular}




\begin{tabular}{|c|c|c|c|c|c|c|c|c|c|}
\hline 1 & 2 & 3 & 4 & 5 & 6 & 7 & 8 & 9 & 10 \\
\hline $\begin{array}{l}\text { 101. Neptis clinia } \\
\text { praedicta Smetacek }\end{array}$ & India to Indo-China. & LO & $\begin{array}{l}\text { TD, } \\
\text { SR }\end{array}$ & $\mathrm{O}$ & 0 to 2 & $\begin{array}{l}\text { M: iii-vi; } \\
\text { x-xi. }\end{array}$ & $?$ & $? P$ & $\begin{array}{l}\text { ?Bombacaceae; } \\
\text { Ulmaceae; etc. }\end{array}$ \\
\hline $\begin{array}{l}\text { 102. Neptis hylas } \\
\text { kamarupa Moore }\end{array}$ & Himalaya & NA & $\begin{array}{l}\text { TD, } \\
\text { SR }\end{array}$ & $\mathrm{O}$ & 1 to 2 & $\begin{array}{l}\text { M: iii-vi; } \\
\text { ix-xi. }\end{array}$ & $?$ & $\mathrm{P}$ & $\begin{array}{l}\text { Leguminosae; } \\
\text { Bombacaceae; } \\
\text { etc. }\end{array}$ \\
\hline $\begin{array}{l}\text { 103. Neptis sappho } \\
\text { astola Moore }\end{array}$ & Palaearctic Region. & NA & $\begin{array}{c}\text { SE, } \\
\text { TD, } \\
\text { SR, S }\end{array}$ & A & 0 to 2 & $\begin{array}{l}\text { M: ii-vi; } \\
\text { ix-xi. }\end{array}$ & $?$ & $\mathrm{O}$ & Leguminosae \\
\hline $\begin{array}{l}\text { 104. Neptis soma } \\
\text { butleri Eliot }\end{array}$ & India to China. & LO & SE & A & 0 to 2 & M: iii; v-x. & $?$ & $\mathrm{O}$ & Ulmaceae \\
\hline $\begin{array}{l}\text { 105. Neptis sankara } \\
\text { sankara Kollar }\end{array}$ & Himalaya to China. & LO & SE & $\mathrm{O}$ & 0 to 2 & B: iv-viii; $x$. & $?$ & $?$ & $?$ \\
\hline $\begin{array}{l}\text { 106. Neptis cartica } \\
\text { cartica Moore }\end{array}$ & Himalaya. & LO & $\begin{array}{l}\text { ?SE/ } \\
\text { ?TD }\end{array}$ & V & $? 0$ to ?2 & S: iv- vi. & $?$ & $?$ & $?$ \\
\hline $\begin{array}{l}\text { 107. Neptis miah } \\
\text { varshneyi Smetacek }\end{array}$ & $\begin{array}{l}\text { West Himalaya to } \\
\text { China. }\end{array}$ & LO & TD & $\mathrm{O}$ & 0 to 2 & $\begin{array}{l}\text { B: iv-vi; } \\
\text { x-xi. }\end{array}$ & $?$ & $?$ & $?$ \\
\hline $\begin{array}{l}\text { 108. Neptis zaida } \\
\text { zaida Westwood }\end{array}$ & $\begin{array}{l}\text { West Himalaya to } \\
\text { China. }\end{array}$ & LO & $\begin{array}{l}\text { ?SE/ } \\
\text { ?TD }\end{array}$ & V & 0 to 1 & S: v-vii. & $?$ & $?$ & $?$ \\
\hline $\begin{array}{l}\text { 109. Neptis ananta } \\
\text { Moore }\end{array}$ & $\begin{array}{l}\text { Himalaya to } \\
\text { Malaysia. }\end{array}$ & LO & $\begin{array}{l}\text { SE/ } \\
\text { TD }\end{array}$ & $\mathrm{R}$ & 1 to 2 & $\begin{array}{l}\text { B: v-vi; } \\
\text { viii-x. }\end{array}$ & $?$ & $\mathrm{O}$ & Lauraceae \\
\hline $\begin{array}{l}\text { 110. Pantoporia } \\
\text { hordonia hordonia } \\
\text { Stoll }\end{array}$ & Oriental Region & RM & $\begin{array}{l}\text { TD, } \\
\text { SR }\end{array}$ & $\mathrm{O}$ & 0 to 2 & $\begin{array}{l}\text { B: iv-vi; } \\
\text { x-xi. }\end{array}$ & $?$ & $\mathrm{O}$ & Leguminosae. \\
\hline $\begin{array}{l}\text { 111. Pantoporia } \\
\text { sandaka Moore }\end{array}$ & Oriental Region & RM & $\begin{array}{l}\text { TD, } \\
\text { SR }\end{array}$ & $\mathrm{O}$ & 0 to 2 & $\begin{array}{l}\text { B: iv-vi; } \\
\text { x-xi. }\end{array}$ & $?$ & $? 0$ & Leguminosae. \\
\hline $\begin{array}{l}\text { 112. Cyrestis } \\
\text { thyodamas ganescha } \\
\text { Kollar }\end{array}$ & India to Japan. & NA & $\begin{array}{l}\text { SE, } \\
\text { TD }\end{array}$ & $\mathrm{O}$ & 0 to 2 & M: ii-x. & $?$ & ?P & $\begin{array}{l}\text { Moraceae; } \\
\text { ?Dilleniaceae; }\end{array}$ \\
\hline $\begin{array}{l}\text { 113. Pseudergolis } \\
\text { wedah Kollar }\end{array}$ & Himalaya to China. & NA & SE & $\mathrm{O}$ & 0 to 2 & M: iii-ix. & $?$ & $\mathrm{O}$ & Urticaceae. \\
\hline $\begin{array}{l}\text { 114. Hypolimnas } \\
\text { bolina Linnaeus }\end{array}$ & $\begin{array}{l}\text { Indo-Australian } \\
\text { Region }\end{array}$ & RM & $\begin{array}{l}\text { TD, } \\
\text { SR }\end{array}$ & $\mathrm{R}$ & 2 to 3 & $\begin{array}{l}\text { M: iii; vii- } \\
\quad \text { xiii. }\end{array}$ & $?$ & $\mathrm{P}$ & $\begin{array}{l}\text { Acanthaceae; } \\
\text { Convolvulaceae; } \\
\text { etc. }\end{array}$ \\
\hline $\begin{array}{l}\text { 115. Hypolimnas } \\
\text { misippus Linnaeus }\end{array}$ & $\begin{array}{l}\text { African, southern } \\
\text { Palaearctic, Oriental, } \\
\text { Australian, southern } \\
\text { Nearctic and } \\
\text { northern Neotropical } \\
\text { Regions }\end{array}$ & SM & SR & V & 2 to 3 & $\begin{array}{l}\text { ?M: iv; } \\
\text { viii-xii. }\end{array}$ & $?$ & $\mathrm{P}$ & $\begin{array}{l}\text { Acanthaceae; } \\
\text { Convolvulaceae; } \\
\text { etc }\end{array}$ \\
\hline $\begin{array}{l}\text { 116. Kallima inachus } \\
\text { huegeli Kollar }\end{array}$ & India to China & NA & $\begin{array}{l}\text { SE, } \\
\text { TD }\end{array}$ & $\mathrm{O}$ & 0 to 2 & M: iv-x. & $?$ & $\mathrm{P}$ & $\begin{array}{l}\text { Urticaceae, } \\
\text { Acanthaceae; etc. }\end{array}$ \\
\hline $\begin{array}{l}\text { 117. Junonia hierta } \\
\text { hierta Fabricius }\end{array}$ & Oriental Region & NA & $\begin{array}{l}\text { TD, } \\
\text { SR, S }\end{array}$ & A & 1 to 3 & M: iii-viii; x. & $?$ & $\mathrm{O}$ & Acanthaceae \\
\hline $\begin{array}{l}\text { 118. Junonia orithya } \\
\text { Linnaeus }\end{array}$ & $\begin{array}{l}\text { African, Palaearctic, } \\
\text { Indo-Australian } \\
\text { Regions }\end{array}$ & NA & $\begin{array}{l}\text { TD, } \\
\text { SR, S }\end{array}$ & A & 1 to 3 & $\begin{array}{l}\text { M: iii-vi; ix- } \\
\quad \text { x; xii-i. }\end{array}$ & $?$ & $\mathrm{P}$ & $\begin{array}{l}\text { Acanthaceae; } \\
\text { Convolvulaceae; } \\
\text { etc. }\end{array}$ \\
\hline $\begin{array}{l}\text { 119. Junonia } \\
\text { lemonias persicaria } \\
\text { Fruhstorfer }\end{array}$ & $\begin{array}{l}\text { India to China and } \\
\text { Malaysia }\end{array}$ & G, NA & $\begin{array}{l}\text { TD, } \\
\text { SR, S }\end{array}$ & A & 0 to 2 & M: i-xi. & $?$ & $\mathrm{P}$ & $\begin{array}{l}\text { Acanthaceae; } \\
\text { Cannabaceae }\end{array}$ \\
\hline $\begin{array}{l}\text { 120. Junonia almana } \\
\text { Linnaeus }\end{array}$ & $\begin{array}{l}\text { India to the } \\
\text { Philippines }\end{array}$ & NA & $\begin{array}{l}\text { TD, } \\
\text { SR, S }\end{array}$ & $\mathrm{O}$ & 0 to 2 & $\begin{array}{l}\text { M: i-vi; } \\
\text { ix-x. }\end{array}$ & $?$ & $\mathrm{P}$ & $\begin{array}{l}\text { Acanthaceae; } \\
\text { Graminae; etc. }\end{array}$ \\
\hline $\begin{array}{l}\text { 121. Junonia atlites } \\
\text { Linnaeus }\end{array}$ & India to Sulawesi & NA & TD & $\mathrm{O}$ & 0 to 2 & $\begin{array}{l}\text { M: iv-vi; } \\
\text { x-xii. }\end{array}$ & $?$ & $\mathrm{P}$ & $\begin{array}{l}\text { Acanthaceae; } \\
\text { Amaranthaceae; } \\
\text { etc. }\end{array}$ \\
\hline $\begin{array}{l}\text { 122. Junonia iphita } \\
\text { siccata Stichel }\end{array}$ & $\begin{array}{l}\text { India to China and } \\
\text { Malaysia }\end{array}$ & NA & $\begin{array}{l}\text { SE, } \\
\text { TD }\end{array}$ & A & 0 to 2 & M: i-x. & $P$ & $\mathrm{O}$ & Acanthaceae \\
\hline $\begin{array}{l}\text { 123. Vanessa cardui } \\
\text { Linnaeus }\end{array}$ & $\begin{array}{l}\text { Nearctic, African, } \\
\text { Oriental, Australian, } \\
\text { Palaearctic Regions }\end{array}$ & NA & $U$ & A & 3 & M: i- xi. & $?$ & $\mathrm{P}$ & $\begin{array}{l}\text { Urticaceae, } \\
\text { Asteraceae, etc. }\end{array}$ \\
\hline $\begin{array}{l}\text { 124. Vanessa indica } \\
\text { indica Herbst }\end{array}$ & India to S.E. Asia. & NA & SE & A & 0 to 3 & M: iii-i. & ?P & $\mathrm{P}$ & $\begin{array}{l}\text { Urticaceae; } \\
\text { Tiliaceae; } \\
\text { Ulmaceae. }\end{array}$ \\
\hline $\begin{array}{l}\text { 125. Kaniska canace } \\
\text { himalaya Evans }\end{array}$ & India to Malaysia. & NA & SE & $\mathrm{O}$ & 0 to 2 & $\begin{array}{l}\text { M: I; v-vii; } \\
\text { ix-xi; }\end{array}$ & $?$ & $\mathrm{P}$ & $\begin{array}{l}\text { Liliaceae, } \\
\text { Smilacaceae, } \\
\text { Dioscoreaceae }\end{array}$ \\
\hline
\end{tabular}




\begin{tabular}{|c|c|c|c|c|c|c|c|c|c|}
\hline 1 & 2 & 3 & 4 & 5 & 6 & 7 & 8 & 9 & 10 \\
\hline $\begin{array}{l}\text { 126. Nymphalis } \\
\text { xanthomelas } \\
\text { fervescens Stichel }\end{array}$ & Palaearctic Region. & SM & SE & $\mathrm{V}$ & 0 to 2 & M: iii; v; ix. & $\mathrm{P}$ & $\mathrm{P}$ & $\begin{array}{l}\text { Ulmaceae; } \\
\text { Salicaceae; } \\
\text { Anacardiaceae. }\end{array}$ \\
\hline $\begin{array}{l}\text { 127. Aglais } \\
\text { cashmirensis aesis } \\
\text { Fruhstorfer }\end{array}$ & Himalaya. & G, NA & SE & $\mathrm{O}$ & 0 to 2 & M: i-xii. & A & $\mathrm{O}$ & Urticaceae \\
\hline $\begin{array}{l}\text { 128. Symbrenthia } \\
\text { lilaea khasiana Moore }\end{array}$ & $\begin{array}{l}\text { India to China and } \\
\text { Malaysia. }\end{array}$ & NA & SE & $\mathrm{O}$ & 0 to 2 & $\begin{array}{l}\text { M: ii-v; } \\
\text { viii-x. }\end{array}$ & $?$ & $\mathrm{O}$ & Urticaceae \\
\hline $\begin{array}{l}\text { 129. Symbrenthia } \\
\text { brabira Moore }\end{array}$ & $\begin{array}{l}\text { Himalaya to W. } \\
\text { China. }\end{array}$ & NA & SE & $\mathrm{V}$ & 0 to 2 & B: v; viii. & $?$ & $?$ & ?Urticaceae \\
\hline $\begin{array}{l}\text { 130. Symbrenthia } \\
\text { niphanda hysudra } \\
\text { Moore }\end{array}$ & Himalaya. & NA & SE & $\mathrm{V}$ & 0 to 2 & ?S: v. & $?$ & $?$ & $?$ \\
\hline $\begin{array}{l}\text { 131. Argynnis } \\
\text { (Argyreus) hyperbius } \\
\text { Linnaeus }\end{array}$ & $\begin{array}{l}\text { Abyssinia through } \\
\text { India to Australia }\end{array}$ & NA & $\begin{array}{l}\mathrm{SE} \\
\mathrm{S}, \mathrm{G}\end{array}$ & $\mathrm{O}$ & 1 to 2 & $\begin{array}{l}\text { M: iv-v; vii- } \\
\text { viii; } x-x i i .\end{array}$ & $?$ & $\mathrm{P}$ & $\begin{array}{l}\text { Violaceae; } \\
\text { Scrophulariaceae }\end{array}$ \\
\hline $\begin{array}{l}\text { 132. Argynnis } \\
\text { (Childrena) childreni } \\
\text { sakontala Kollar }\end{array}$ & Himalaya to China & NA/?RM & SE & V & 1 to 2 & $B: v-x$ & $?$ & $?$ & $?$ \\
\hline $\begin{array}{l}\text { 133. Issoria lathonia } \\
\text { issaea Doubleday }\end{array}$ & Palaearctic Region. & SM & SE, G & V & 0 to 2 & M: iii-x. & $?$ & $\mathrm{O}$ & Violaceae. \\
\hline $\begin{array}{l}\text { 134. Cupha } \\
\text { erymanthis lotis Sulz }\end{array}$ & India to Malaysia. & RM & TD & $A$ & 2 to 3 & $\begin{array}{l}\text { B: iv-vi; } \\
\text { viii-x. }\end{array}$ & $?$ & $\mathrm{P}$ & $\begin{array}{l}\text { Flacourtiaceae; } \\
\text { Sapindaceae. }\end{array}$ \\
\hline $\begin{array}{l}\text { 135. Phalanta } \\
\text { phalantha Drury }\end{array}$ & $\begin{array}{l}\text { India to Japan and } \\
\text { Australia. }\end{array}$ & $\mathrm{G}, \mathrm{NA}$ & $\begin{array}{l}\text { TD, } \\
\text { SR }\end{array}$ & $C$ & 0 to 3 & M: v-x. & $?$ & $\mathrm{P}$ & $\begin{array}{l}\text { Salicaceae; } \\
\text { Flacourtiaceae; } \\
\text { etc. }\end{array}$ \\
\hline $\begin{array}{l}\text { 136. Vagrans sinha } \\
\text { pallida Evans }\end{array}$ & $\begin{array}{l}\text { Indo-Australian } \\
\text { Region. }\end{array}$ & NA/?RM & $\begin{array}{l}\text { TD, } \\
\text { SR }\end{array}$ & $\mathrm{O}$ & 1 to 2 & M: v; vii-x. & $?$ & $\mathrm{P}$ & $\begin{array}{l}\text { Flacourtiaceae; } \\
\text { Dilleniaceae. }\end{array}$ \\
\hline $\begin{array}{l}\text { 137. Ariadne merione } \\
\text { tapestrina Moore }\end{array}$ & India to Malaysia. & NA/G & $\begin{array}{l}\text { TD, } \\
\text { SR }\end{array}$ & $C$ & 0 to 2 & M: iii; v-xii. & $? \mathrm{P}$ & $\mathrm{O}$ & Euphorbiaceae \\
\hline $\begin{array}{l}\text { 138. Acraea issoria } \\
\text { anomala Kollar }\end{array}$ & Himalaya to China. & NA & SE & $C$ & 0 to 1 & M: iv-ix. & ?P & $\mathrm{P}$ & $\begin{array}{l}\text { Rubiaceae; } \\
\text { Urticaceae }\end{array}$ \\
\hline $\begin{array}{l}\text { 139. Acraea violae } \\
\text { Fabricius }\end{array}$ & India to Sri Lanka. & SM & SR, S & V & 1 to 2 & $\begin{array}{l}\text { M: iv; vii- } \\
\text { viii; } x \text {. }\end{array}$ & $?$ & $\mathrm{P}$ & $\begin{array}{l}\text { Malvaceae; } \\
\text { Passifloraceae, } \\
\text { etc. }\end{array}$ \\
\hline $\begin{array}{l}\text { 140. Libythea lepita } \\
\text { lepita Moore }\end{array}$ & India to China. & NA & SE & $\mathrm{O}$ & 0 to 1 & M: i-xi. & $?$ & $\mathrm{O}$ & Ulmaceae \\
\hline $\begin{array}{l}\text { 141. Libythea } \\
\text { myrrha sanguinalis } \\
\text { Fruhstorfer }\end{array}$ & India to China. & NA & SE & $\mathrm{O}$ & 0 to 1 & M: ii-x. & $?$ & $\mathrm{O}$ & Ulmaceae \\
\hline \multicolumn{10}{|l|}{ LYCAENIDAE } \\
\hline $\begin{array}{l}\text { 142. Zemeros flegyas } \\
\text { indicus Fruhstorfer }\end{array}$ & $\begin{array}{l}\text { Himalaya to China } \\
\text { and Malaysia. }\end{array}$ & SM & $\begin{array}{l}\text { TD, } \\
\text { SR }\end{array}$ & $\mathrm{V}$ & 0 to 2 & M: iii-x. & $?$ & $\mathrm{O}$ & Myrsinaceae. \\
\hline $\begin{array}{l}\text { 143. Dodona durga } \\
\text { Kollar }\end{array}$ & Himalaya to China. & SM & SE & $\mathrm{R}$ & 0 to 2 & M: iii-xi. & $?$ & $\mathrm{O}$ & Graminae \\
\hline $\begin{array}{l}\text { 144. Dodona } \\
\text { eugenes Bates }\end{array}$ & Himalaya to China. & SM & SE & V & 0 to 2 & M: iii-x. & $?$ & $\mathrm{P}$ & $\begin{array}{l}\text { Myrsinaceae; } \\
\text { Graminae }\end{array}$ \\
\hline $\begin{array}{l}\text { 145. Dodona dipoea } \\
\text { nostia Fruhstorfer }\end{array}$ & Himalaya & SM & SE & V & 0 to 2 & M: iii-xi. & $?$ & $\mathrm{O}$ & Myrsinaceae \\
\hline $\begin{array}{l}\text { 146. Abisara echerius } \\
\text { suffusa Moore }\end{array}$ & India to China. & LO & $\begin{array}{l}\text { TD, } \\
\text { SR }\end{array}$ & $\mathrm{R}$ & 0 to 2 & $\begin{array}{l}\text { M: iv-v; } \\
\text { vii-x. }\end{array}$ & $?$ & $?$ & Myrsinaceae \\
\hline $\begin{array}{l}\text { 147. Abisara fylla } \\
\text { Doubleday }\end{array}$ & Himalaya to China. & LO & SE & $\mathrm{R}$ & 1 to 2 & B: iii-v; ix. & $?$ & $?$ & Myrsinaceae \\
\hline $\begin{array}{l}\text { 148. Poritia hewitsoni } \\
\text { hewitsoni Moore }\end{array}$ & Himalaya & SM & SR & V & 1 to 2 & M: iii; v; ix. & $?$ & $\mathrm{O}$ & Dipterocarpaceae \\
\hline $\begin{array}{l}\text { 149. Miletus } \\
\text { chinensis assamensis } \\
\text { Doherty }\end{array}$ & Himalaya to China. & LO & SE & V & 0 to 2 & ?M: ix. & $?$ & $\begin{array}{l}\text { Inse- } \\
\text { cta }\end{array}$ & Aphididae \\
\hline $\begin{array}{l}\text { 150. Allotinus drumila } \\
\text { Moore }\end{array}$ & $\begin{array}{l}\text { Himalaya to } \\
\text { Myanmar. }\end{array}$ & LO & $?$ & V & $?$ & $?$ & $?$ & $\begin{array}{l}\text { Inse- } \\
\text { cta }\end{array}$ & $?$ \\
\hline $\begin{array}{l}\text { 151. Talicada nyseus } \\
\text { Guérin-Menéville }\end{array}$ & India to Thailand & LO & $\begin{array}{l}\text { TD, } \\
\text { SR }\end{array}$ & $\mathrm{R}$ & 0 to 2 & M: i - xi.. & $?$ & $\mathrm{O}$ & Crassulaceae \\
\hline $\begin{array}{l}\text { 152. Chilades laius } \\
\text { laius Cramer }\end{array}$ & $\begin{array}{l}\text { India to China, S.E. } \\
\text { Asia }\end{array}$ & G; NA. & $\begin{array}{l}\text { TD; } \\
\text { SR }\end{array}$ & A & 0 to 2 & M: xi-iii & $?$ & $\mathrm{P}$ & $\begin{array}{l}\text { Rutaceae; } \\
\text { Anacardiaceae; } \\
\text { etc. }\end{array}$ \\
\hline
\end{tabular}




\begin{tabular}{|c|c|c|c|c|c|c|c|c|c|}
\hline 1 & 2 & 3 & 4 & 5 & 6 & 7 & 8 & 9 & 10 \\
\hline $\begin{array}{l}\text { 153. Chilades } \\
\text { pandava Horsfield }\end{array}$ & India to Sumatra. & NA. & $\begin{array}{l}\text { SE; } \\
\text { TD. }\end{array}$ & $\mathrm{O}$ & 0 to 2 & M: vi-x. & $?$ & $\mathrm{P}$ & $\begin{array}{l}\text { Rutaceae; } \\
\text { Leguminosae; etc. }\end{array}$ \\
\hline $\begin{array}{l}\text { 154. Neopithecops } \\
\text { zalmora Butler }\end{array}$ & India to Australia. & NA. & TD. & $\mathrm{O}$ & 0 to 2 & B: iii; vii. & $?$ & $\mathrm{P}$ & $\begin{array}{l}\text { Rutaceae; } \\
\text { Ebenaceae. }\end{array}$ \\
\hline $\begin{array}{l}\text { 155. Everes argiades } \\
\text { indica Evans }\end{array}$ & Himalaya & NA. & SE. & $\mathrm{O}$ & 1 to 2 & M: iv; vii-ix. & $?$ & $\mathrm{O}$ & Leguminosae \\
\hline $\begin{array}{l}\text { 156. Everes diporides } \\
\text { Chapman }\end{array}$ & $\begin{array}{l}\text { Himalaya to Indo- } \\
\text { China. }\end{array}$ & NA. & SE. & $\mathrm{O}$ & 1 to 2 & M: v-x. & $?$ & $?$ & $?$ \\
\hline $\begin{array}{l}\text { 157. Everes huegelii } \\
\text { dipora Moore }\end{array}$ & $\begin{array}{l}\text { Himalaya to Indo- } \\
\text { China. }\end{array}$ & NA. & SE. & $\mathrm{O}$ & 1 to 2 & M: iv; ix. & $?$ & $?$ & Leguminosae \\
\hline $\begin{array}{l}\text { 158. Megisba malaya } \\
\text { sikkima Moore }\end{array}$ & $\begin{array}{l}\text { Himalaya to Taiwan } \\
\text { and Sulawesi. }\end{array}$ & NA. & $\begin{array}{l}\text { SE; } \\
\text { TD; } \\
\text { SR. }\end{array}$ & $A$ & 1 to 2 & B: vii; $x$. & $?$ & $\mathrm{P}$ & $\begin{array}{l}\text { Euphorbiaceae; } \\
\text { Sapindaceae; etc. }\end{array}$ \\
\hline $\begin{array}{l}\text { 159. Acytolepis puspa } \\
\text { gisca Fruhstorfer }\end{array}$ & $\begin{array}{l}\text { India to Japan and } \\
\text { the Philippines. }\end{array}$ & G; NA. & $\begin{array}{l}\text { SE; } \\
\text { SR. }\end{array}$ & $\mathrm{O}$ & 0 to 2 & M: vii-x. & $?$ & $\mathrm{P}$ & $\begin{array}{l}\text { Fagaceae; } \\
\text { Ericaceae, etc. }\end{array}$ \\
\hline $\begin{array}{l}\text { 160. Udara dilecta } \\
\text { dilecta Moore }\end{array}$ & $\begin{array}{l}\text { Pakistan to Japan } \\
\text { and Malaysia. }\end{array}$ & NA. & SE. & $\mathrm{R}$ & 2 & M: v; viii; $x$. & $?$ & $\mathrm{O}$ & Fagaceae. \\
\hline $\begin{array}{l}\text { 161. Celastrina } \\
\text { huegelii huegelii } \\
\text { Moore }\end{array}$ & $\begin{array}{l}\text { West Himalaya to } \\
\text { China. }\end{array}$ & NA. & SE. & $\mathrm{C}$ & 0 to 1 & M: iv-x. & $?$ & $? 0$ & Rosaceae \\
\hline $\begin{array}{l}\text { 162. Celastrina gigas } \\
\text { Hemming }\end{array}$ & $\begin{array}{l}\text { West Himalayan } \\
\text { endemic. }\end{array}$ & NA. & SE. & C & 0 to 1 & M: iv- $x$ & $?$ & $\mathrm{M}$ & Rosaceae \\
\hline $\begin{array}{l}\text { 163. Celastrina } \\
\text { argiolus kollari } \\
\text { Westwood }\end{array}$ & Palaearctic Region. & G; NA. & SE. & $\mathrm{C}$ & 1 to 2 & M: iv-x. & $?$ & $? \mathrm{P}$ & $\begin{array}{l}\text { Leguminosae; } \\
\text { Rosaceae; etc. }\end{array}$ \\
\hline $\begin{array}{l}\text { 164. Aricia } \\
\text { agestis Denis \& } \\
\text { Schiffermuller }\end{array}$ & Palaearctic Region.. & NA. & SE. & $\mathrm{O}$ & 0 to 2 & M: iii-vii. & $?$ & $?$ & $?$ \\
\hline $\begin{array}{l}\text { 165. Leptotes plinius } \\
\text { Fabricius }\end{array}$ & India to Australia & $\begin{array}{l}\text { G; NA; } \\
\text { RM. }\end{array}$ & U. & A & $2-3$ & $\begin{array}{l}\text { M: iv; vii } \\
\quad \text {-ix.. }\end{array}$ & $?$ & $\mathrm{P}$ & $\begin{array}{l}\text { Leguminosae; } \\
\text { Rhamnaceae; etc. }\end{array}$ \\
\hline $\begin{array}{l}\text { 166. Freyeria } \\
\text { trochylus Freyer }\end{array}$ & India to Sumatra. & NA. & $\begin{array}{l}\text { TD; } \\
\text { SR; } \\
\text { S; G. }\end{array}$ & $\mathrm{O}$ & 1 to 2 & $M: x$ & $?$ & $\mathrm{P}$ & $\begin{array}{l}\text { Boraginaceae; } \\
\text { Leguminosae; etc. }\end{array}$ \\
\hline $\begin{array}{l}\text { 167. Pseudozizeeria } \\
\text { maha maha Kollar }\end{array}$ & $\begin{array}{l}\text { Iran to India and } \\
\text { Japan. }\end{array}$ & G; NA. & $\begin{array}{l}\text { TD; } \\
\text { SR; } \\
\text { S; G. }\end{array}$ & $\mathrm{C}$ & 0 to 1 & M: vii-x. & $?$ & $\mathrm{P}$ & $\begin{array}{l}\text { Oxalidaceae; } \\
\text { Acanthaceae; etc. }\end{array}$ \\
\hline $\begin{array}{l}\text { 168. Zizeeria } \\
\text { karsandra Moore }\end{array}$ & $\begin{array}{l}\text { N. Africa through } \\
\text { Asia to Australia. }\end{array}$ & G; NA. & $\begin{array}{l}\text { TD; } \\
\text { SE; } \\
\text { SR; } \\
\text { S: G. }\end{array}$ & $\mathrm{O}$ & 1 to 2 & M: iv-ix. & $?$ & $\mathrm{P}$ & $\begin{array}{l}\text { Leguminosae; } \\
\text { Amaranthaceae. }\end{array}$ \\
\hline $\begin{array}{l}\text { 169. Zizula hylax } \\
\text { Fabricius }\end{array}$ & $\begin{array}{l}\text { African Region, N. } \\
\text { America, Asia south } \\
\text { of the Himalaya. }\end{array}$ & G; NA. & $\begin{array}{l}\text { TD; } \\
\text { SR; } \\
\text { S; G. }\end{array}$ & $\mathrm{O}$ & 1 to 2 & B: iv; viii. & $?$ & $\mathrm{P}$ & $\begin{array}{l}\text { Acanthaceae; } \\
\text { Leguminosae; } \\
\text { Graminae; etc. }\end{array}$ \\
\hline $\begin{array}{l}\text { 170. Zizina otis otis } \\
\text { Fabricius }\end{array}$ & $\begin{array}{l}\text { India to China, } \\
\text { Taiwan and Australia. }\end{array}$ & NA. & $\begin{array}{l}\text { TD; } \\
\text { SR; } \\
\text { S; G. }\end{array}$ & $\mathrm{O}$ & 1 to 2 & M: vii-x. & $?$ & $\mathrm{P}$ & $\begin{array}{l}\text { Leguminosae; } \\
\text { Zygophyllaceae. }\end{array}$ \\
\hline $\begin{array}{l}\text { 171. Euchrysops } \\
\text { cnejus Fabricius }\end{array}$ & $\begin{array}{l}\text { India to China and } \\
\text { Australia. }\end{array}$ & $\begin{array}{l}\text { G; NA; } \\
\text { RM. }\end{array}$ & $U$ & C & 3 & M: iv-x. & $?$ & $\mathrm{P}$ & $\begin{array}{l}\text { Leguminosae; } \\
\text { Orchidaceae; etc. }\end{array}$ \\
\hline $\begin{array}{l}\text { 172. Catochrysops } \\
\text { strabo Fabricius }\end{array}$ & India to Moluccas & $\begin{array}{l}\text { G; NA; } \\
\text { RM. }\end{array}$ & $U$ & C & 2 & M: iv-x. & $?$ & $\mathrm{P}$ & $\begin{array}{l}\text { Leguminosae; } \\
\text { Sapindaceae }\end{array}$ \\
\hline $\begin{array}{l}\text { 173. Lampides } \\
\text { boeticus Linnaeus }\end{array}$ & $\begin{array}{l}\text { African, Palaearctic } \\
\text { and Indo-Australian } \\
\text { Regions. }\end{array}$ & $\begin{array}{l}\text { G; NA; } \\
\text { RM. }\end{array}$ & $U$ & $\mathrm{C}$ & 3 & M: ii-x. & $?$ & $\mathrm{P}$ & $\begin{array}{l}\text { Leguminosae; } \\
\text { Liliaceae. }\end{array}$ \\
\hline $\begin{array}{l}\text { 174. Jamides bochus } \\
\text { bochus Cramer }\end{array}$ & India to Australia. & NA; RM. & $\begin{array}{l}\text { TD; } \\
\text { SR. }\end{array}$ & $\mathrm{O}$ & 1 to 2 & B: iv; $x$. & $?$ & $\mathrm{O}$ & Leguminosae. \\
\hline $\begin{array}{l}\text { 175. Jamides celeno } \\
\text { celeno Cramer }\end{array}$ & India to Sulawesi. & NA. & $\begin{array}{l}\text { TD; } \\
\text { SR. }\end{array}$ & $\mathrm{O}$ & 0 to 2 & M: iv- $x$ & $?$ & $\mathrm{P}$ & $\begin{array}{l}\text { Leguminosae; } \\
\text { Zingiberaceae; etc. }\end{array}$ \\
\hline $\begin{array}{l}\text { 176. Prosotas nora } \\
\text { nora Felder \& Felder }\end{array}$ & India to Myanmar. & NA. & $\begin{array}{l}\text { TD; } \\
\text { SR. }\end{array}$ & $\mathrm{O}$ & 0 to 1 & M: i-xii. & $?$ & $\mathrm{P}$ & $\begin{array}{l}\text { Leguminosae; } \\
\text { Combretaceae; } \\
\text { etc. }\end{array}$ \\
\hline $\begin{array}{l}\text { 177. Prosotas noreia } \\
\text { hampsoni de Nicéville }\end{array}$ & India to Myanmar. & NA. & $\begin{array}{l}\text { TD; } \\
\text { SR. }\end{array}$ & $\mathrm{R}$ & 0 to 1 & M : vii. & $?$ & $?$ & $?$ \\
\hline $\begin{array}{l}\text { 178. Lycaena pavana } \\
\text { Kollar }\end{array}$ & $\begin{array}{l}\text { West Himalayan } \\
\text { endemic. }\end{array}$ & NA; LO. & $\begin{array}{l}\text { S ; } \\
\text { SE. }\end{array}$ & $\mathrm{R}$ & 0 to 1 & M : i ; iv-vi. & $?$ & $?$ & ?Polygonaceae \\
\hline
\end{tabular}




\begin{tabular}{|c|c|c|c|c|c|c|c|c|c|}
\hline 1 & 2 & 3 & 4 & 5 & 6 & 7 & 8 & 9 & 10 \\
\hline $\begin{array}{l}\text { 179. Lycaena phlaeas } \\
\text { indicus Evans }\end{array}$ & $\begin{array}{l}\text { Palaearctic and } \\
\text { Nearctic Regions }\end{array}$ & NA. & $\begin{array}{l}\text { S ; } \\
\text { SE. }\end{array}$ & 0 & 0 to 1 & $M:$ ii-x. & $?$ & $P$ & $\begin{array}{l}\text { Polygonaceae; } \\
\text { Malvaceae. }\end{array}$ \\
\hline $\begin{array}{l}\text { 180. Heliophorus } \\
\text { sena Kollar }\end{array}$ & Himalayan. & $\mathrm{G} ; \mathrm{NA}$. & $\begin{array}{l}\text { S ; } \\
\text { SE. }\end{array}$ & $A$ & 0 to 2 & M : iv-x. & $?$ & $\mathrm{P}$ & $\begin{array}{l}\text { Polygonaceae; } \\
\text { Malvaceae. }\end{array}$ \\
\hline $\begin{array}{l}\text { 181. Heliophorus } \\
\text { moorei coruscans } \\
\text { Moore }\end{array}$ & $\begin{array}{l}\text { Himalaya to } \\
\text { W.China. }\end{array}$ & SM. & $\begin{array}{l}\text { S; } \\
\text { SE. }\end{array}$ & $\mathrm{V}$ & 0 to 2 & B : v-iv ; ix. & $?$ & $?$ & $?$ \\
\hline $\begin{array}{l}\text { 182. Pamela } \\
\text { dudgeoni de Nicéville }\end{array}$ & $\begin{array}{l}\text { Himalaya to Indo- } \\
\text { China. }\end{array}$ & LO. & SE. & $\mathrm{V}$ & $?$ & $B$ : iv ; $x$ & $?$ & $?$ & $?$ \\
\hline $\begin{array}{l}\text { 183. Thermozephyrus } \\
\text { ataxus ataxus } \\
\text { Doubleday }\end{array}$ & Himalaya to China. & LO. & SE. & $\mathrm{V}$ & 0 to 1 & $M: v-x$ & $?$ & $? 0$ & Fagaceae \\
\hline $\begin{array}{l}\text { 184. Chrysozephyrus } \\
\text { syla syla Kollar }\end{array}$ & Himalaya. & NA; LO. & SE. & $\mathrm{R}$ & 0 to 2 & $\begin{array}{l}\text { B : iv-vi } \\
\text { ix-x. }\end{array}$ & $?$ & $?$ & $?$ \\
\hline $\begin{array}{l}\text { 185. Euaspa ziha de } \\
\text { Nicéville }\end{array}$ & $\begin{array}{l}\text { West Himalayan } \\
\text { endemic. }\end{array}$ & NA; LO. & SE. & $A$ & 0 to 1 & S : v-vi. & $?$ & $?$ & $?$ \\
\hline $\begin{array}{l}\text { 186. Curetis bulis } \\
\text { Doubleday \& } \\
\text { Hewitson }\end{array}$ & India to Japan & $\mathrm{G}$; NA. & $\begin{array}{l}\text { TD } \\
\text { SR. }\end{array}$ & $\mathrm{R}$ & 0 to 2 & $M: v-x$ & $?$ & $\mathrm{O}$ & Leguminosae \\
\hline $\begin{array}{l}\text { 187. Curetis acuta } \\
\text { dentata Moore }\end{array}$ & India to S.E. Asia. & G; NA. & $\begin{array}{l}\text { TD; } \\
\text { SR. }\end{array}$ & $\mathrm{R}$ & 0 to 2 & $M: v-x$ & $?$ & $\mathrm{O}$ & Leguminosae \\
\hline $\begin{array}{l}\text { 188. Iraota timoleon } \\
\text { Stoll }\end{array}$ & India to China. & NA; ?RM. & $\begin{array}{l}\text { TD; } \\
\text { SR. }\end{array}$ & $\mathrm{O}$ & 0 to 2 & $\begin{array}{l}M: v ; \\
\text { vii } ; x\end{array}$ & $?$ & $P$ & $\begin{array}{l}\text { Lythraceae; } \\
\text { Moraceae; }\end{array}$ \\
\hline $\begin{array}{l}\text { 189. Arhopala atrax } \\
\text { Hewitson }\end{array}$ & India to Malaysia. & RM. & SR. & $\mathrm{O}$ & 0 to 2 & S : vi. & $?$ & M & Dipterocarpaceae \\
\hline $\begin{array}{l}\text { 190. Arhopala singla } \\
\text { de Nicéville }\end{array}$ & $\begin{array}{l}\text { Himalaya to S.W. } \\
\text { China. }\end{array}$ & SM. & $?$ & V & $?$ & $S: v$ & $?$ & $?$ & $?$ \\
\hline $\begin{array}{l}\text { 191. Arhopala } \\
\text { dodonea Moore }\end{array}$ & $\begin{array}{l}\text { West Himalayan } \\
\text { endemic. }\end{array}$ & NA; LO. & SE. & 0 & 0 to 2 & $\begin{array}{l}\mathrm{M}: \mathrm{v}-\mathrm{vi} \\
\mathrm{x} ; \mathrm{xii-i}\end{array}$ & $?$ & M & Fagaceae \\
\hline $\begin{array}{l}\text { 192. Arhopala rama } \\
\text { rama Kollar }\end{array}$ & Himalaya & NA. & $\begin{array}{l}\text { SE ; } \\
\text { SR. }\end{array}$ & 0 & 0 to 2 & $\begin{array}{l}\text { M : iii-vi } \\
\quad x ; \text { xii-i. }\end{array}$ & $?$ & $\mathrm{O}$ & $\begin{array}{l}\text { Fagaceae; } \\
\text { Dipterocarpaceae }\end{array}$ \\
\hline $\begin{array}{l}\text { 193. Arhopala ganesa } \\
\text { ganesa Moore }\end{array}$ & Himalaya to Japan & NA. & SE. & A & 0 to 2 & S : v-vi. & $?$ & $\mathrm{O}$ & Fagaceae \\
\hline $\begin{array}{l}\text { 194. Surendra } \\
\text { vivarna Horsfield }\end{array}$ & India to Sulawesi. & NA. & TD. & 0 & 0 to 2 & M : vii. & $?$ & $\mathrm{O}$ & Leguminosae. \\
\hline $\begin{array}{l}\text { 195. Loxura atymnus } \\
\text { Cramer }\end{array}$ & India to Philippines & NA; ?RM. & $\begin{array}{l}\text { TD } \\
\text { SR. }\end{array}$ & 0 & 1 to 2 & $M$ : iv-x. & $?$ & $\mathrm{P}$ & $\begin{array}{l}\text { Dioscoreaceae; } \\
\text { Smilacaceae }\end{array}$ \\
\hline $\begin{array}{l}\text { 196. Cigaritis } \\
\text { nipalicus Moore }\end{array}$ & Himalayan. & NA; LO. & SE. & $C$ & 0 to 2 & $\begin{array}{l}\text { B : iv-v; } \\
\text { viii. }\end{array}$ & $?$ & $?$ & $?$ \\
\hline $\begin{array}{l}\text { 197. Pratapa deva lila } \\
\text { Moore }\end{array}$ & Himalaya to Java. & LO. & $\begin{array}{l}\text { TD } \\
\text { SE. }\end{array}$ & V & 0 to 2 & S: vii- viii. & $?$ & $\mathrm{O}$ & Loranthaceae \\
\hline $\begin{array}{l}\text { 198. Tajuria diaeus } \\
\text { Hewitson }\end{array}$ & $\begin{array}{l}\text { Himalaya to Indo- } \\
\text { China. }\end{array}$ & NA; LO. & SE. & $\mathrm{V}$ & $?$ & & $?$ & $\mathrm{O}$ & Loranthaceae. \\
\hline $\begin{array}{l}\text { 199. Tajuria illurgis } \\
\text { Hewitson }\end{array}$ & $\begin{array}{l}\text { Himalaya to Indo- } \\
\text { China }\end{array}$ & NA; LO. & SE. & $\mathrm{R}$ & 1 to 2 & $\begin{array}{l}\text { B : iii-iv ; } \\
\text { vi-viii. }\end{array}$ & $?$ & $? 0$ & Loranthaceae. \\
\hline $\begin{array}{l}\text { 200. Horaga onyx } \\
\text { onyx Moore }\end{array}$ & India to Malaysia. & NA; LO. & SE. & $\mathrm{R}$ & 1 to 2 & $B$ : iv ; ix-x. & $?$ & $? 0$ & $\begin{array}{l}\text { Coriariaceae; } \\
\text { Bombacaceae. }\end{array}$ \\
\hline $\begin{array}{l}\text { 201. Horaga } \\
\text { albimacula viola } \\
\text { Moore }\end{array}$ & India to S.E. Asia. & NA; LO. & SE. & $\mathrm{V}$ & 1 to 2 & $B: x$ & $?$ & ?P & Coriariaceae. \\
\hline $\begin{array}{l}\text { 202. Chliaria kina } \\
\text { kina Hewitson }\end{array}$ & $\begin{array}{l}\text { Himalaya to Indo- } \\
\text { China. }\end{array}$ & NA; LO. & SE. & $\mathrm{R}$ & 0 to 2 & $M$ : iv-x. & $?$ & $\mathrm{O}$ & Orchidaceae \\
\hline $\begin{array}{l}\text { 203. Chliaria othona } \\
\text { Hewitson }\end{array}$ & $\begin{array}{l}\text { Himalaya to Indo- } \\
\text { China. }\end{array}$ & NA; LO. & TD. & V & 0 to 2 & S : iv-v. & $?$ & $\mathrm{O}$ & Orchidaceae \\
\hline $\begin{array}{l}\text { 204. Deudorix } \\
\text { epijarbas ancus } \\
\text { Fruhstorfer }\end{array}$ & India to Australia & G ; NA. & $\begin{array}{l}\text { TD } \\
\text { SR. }\end{array}$ & 0 & 0 to 2 & $M: v-x$ & $?$ & $P$ & $\begin{array}{l}\text { Lythraceae; } \\
\text { Sapindaceae; etc. }\end{array}$ \\
\hline $\begin{array}{l}\text { 205. Deudorix } \\
\text { isocrates Fabricius }\end{array}$ & India to Myanmar. & G ; NA. & $\begin{array}{l}\text { TD } \\
\text { SR. }\end{array}$ & 0 & 0 to 2 & B : vii-ix. & $?$ & $\mathrm{P}$ & $\begin{array}{l}\text { Euphorbiaceae ; } \\
\text { Lythraceae; etc. }\end{array}$ \\
\hline $\begin{array}{l}\text { 206. Rapala varuna } \\
\text { grisea Moore }\end{array}$ & India to Australia. & NA; LO. & SE. & A & 0 to 2 & $\begin{array}{l}\text { B : iv-vi } \\
\text { ix-x. }\end{array}$ & $?$ & $\mathrm{P}$ & $\begin{array}{l}\text { Combretaceae; } \\
\text { Myrtaceae; } \\
\text { Sapindaceae; etc. }\end{array}$ \\
\hline $\begin{array}{l}\text { 207. Rapala manea } \\
\text { schistacea Moore }\end{array}$ & India to S.E. Asia. & NA. & $\begin{array}{l}\text { SE; } \\
\text { TD. }\end{array}$ & A & 0 to 2 & M : iv-xi. & $?$ & $P$ & $\begin{array}{l}\text { Caprifoliaceae; } \\
\text { Leguminosae; etc. }\end{array}$ \\
\hline
\end{tabular}




\begin{tabular}{|c|c|c|c|c|c|c|c|c|c|}
\hline 1 & 2 & 3 & 4 & 5 & 6 & 7 & 8 & 9 & 10 \\
\hline $\begin{array}{l}\text { 208. Rapala } \\
\text { pheretima petosiris } \\
\text { Hewitson }\end{array}$ & Himalaya to Borneo. & LO. & $\begin{array}{l}\text { TD } \\
\text { SR. }\end{array}$ & $\mathrm{O}$ & 0 to 2 & B: iv- v. & $?$ & $\mathrm{P}$ & $\begin{array}{l}\text { Anacardiaceae; } \\
\text { Myrtaceae; etc. }\end{array}$ \\
\hline $\begin{array}{l}\text { 209. Rapala iarbus } \\
\text { Fruhstorfer }\end{array}$ & $\begin{array}{l}\text { India to the Sunda } \\
\text { Islands. }\end{array}$ & NA. & $\begin{array}{l}\text { TD; } \\
\text { SR. }\end{array}$ & $\mathrm{O}$ & 0 to 2 & $\begin{array}{l}\text { M : iv-v } \\
\text { vii-xi. }\end{array}$ & $?$ & $\mathrm{P}$ & $\begin{array}{l}\text { Anacardiaceae; } \\
\text { Leguminosae; etc. }\end{array}$ \\
\hline $\begin{array}{l}\text { 210. Rapala nissa } \\
\text { Kollar }\end{array}$ & $\begin{array}{l}\text { Himalaya to China, } \\
\text { Malaysia. }\end{array}$ & NA. & SE. & A & 0 to 2 & M : v-xii. & $?$ & $\mathrm{P}$ & $\begin{array}{l}\text { Leguminosae; } \\
\text { Rosaceae; etc. }\end{array}$ \\
\hline $\begin{array}{l}\text { 211. Sinthusa } \\
\text { chandrana Moore }\end{array}$ & Himalaya. & NA; LO. & SE. & V & $?$ & B : iv ; ix. & $?$ & $\mathrm{O}$ & Rosaceae. \\
\hline $\begin{array}{l}\text { 212. Sinthusa nasaka } \\
\text { pallidior Fruhstorfer }\end{array}$ & $\begin{array}{l}\text { Himalaya to } \\
\text { Malaysia. }\end{array}$ & NA ; LO. & SE. & V & $?$ & S : iv-v. & $?$ & $?$ & $?$ \\
\hline \multicolumn{10}{|l|}{ HESPERIIDAE } \\
\hline $\begin{array}{l}\text { 213. Hasora chromus } \\
\text { chromus Cramer }\end{array}$ & India to Australia. & NA. & $\begin{array}{l}\text { SR ; } \\
\text { TD. }\end{array}$ & $\mathrm{R}$ & 2 & $\begin{array}{l}\text { B : vi } \\
\text { viii-ix. }\end{array}$ & $?$ & $\mathrm{P}$ & $\begin{array}{l}\text { Euphorbiaceae; } \\
\text { Leguminosae; etc. }\end{array}$ \\
\hline $\begin{array}{l}\text { 214. Bibasis } \\
\text { oedipodea belesis } \\
\text { Mabille }\end{array}$ & $\begin{array}{l}\text { Himalaya to China } \\
\text { and Indo-China }\end{array}$ & NA ; ?SM. & TD. & V & 2 to 3 & B : i ; iii. & $?$ & $?$ & Combretaceae \\
\hline $\begin{array}{l}\text { 215. Bibasis anadi de } \\
\text { Nicéville }\end{array}$ & $\begin{array}{l}\text { Himalaya to Indo- } \\
\text { China }\end{array}$ & NA; LO. & SE. & A & 0 & $\begin{array}{l}\text { B: v-vi } \\
\text { viii-ix. }\end{array}$ & $?$ & $?$ & $?$ \\
\hline $\begin{array}{l}\text { 216. Bibasis sena } \\
\text { Moore }\end{array}$ & $\begin{array}{l}\text { India to the } \\
\text { Philippines. }\end{array}$ & NA; LO. & SE. & $\mathrm{R}$ & $? 2$ & $M: v-i x$ & $?$ & $?$ & $\begin{array}{l}\text { Combretaceae; } \\
\text { Malphigiaceae }\end{array}$ \\
\hline $\begin{array}{l}\text { 217. Badamia } \\
\text { exclamationis } \\
\text { Fabricius }\end{array}$ & India to Australia. & NA. & $\begin{array}{l}\text { SR ; } \\
\text { TD. }\end{array}$ & $\mathrm{R}$ & 3 & $\begin{array}{l}\text { B : iv ; } \\
\text { viii-ix. }\end{array}$ & $?$ & $\mathrm{P}$ & $\begin{array}{l}\text { Combretaceae; } \\
\text { Moraceae; etc. }\end{array}$ \\
\hline $\begin{array}{l}\text { 218. Choaspes } \\
\text { benjaminii japonica } \\
\text { Murray }\end{array}$ & $\begin{array}{l}\text { Himalaya to China } \\
\text { and Japan. }\end{array}$ & NA; ?SM. & SE. & V & 2 & M : iii - ix.. & $?$ & $\mathrm{P}$ & $\begin{array}{l}\text { Sabiaceae; } \\
\text { Meliosmaceae. }\end{array}$ \\
\hline $\begin{array}{l}\text { 219. Celaenorrhinus } \\
\text { pero pero de Nicéville }\end{array}$ & Himalaya. & NA ; LO. & SE. & $\mathrm{R}$ & 0 to 2 & $\begin{array}{l}\text { B : iv-vi ; } \\
\text { viii. }\end{array}$ & $?$ & $?$ & $?$ \\
\hline $\begin{array}{l}\text { 220. Celaenorrhinus } \\
\text { leucocera leucocera } \\
\text { Kollar }\end{array}$ & India to Indo-China. & NA. & SE. & A & 0 to 2 & M : iv-x. & $?$ & $\mathrm{O}$ & Acanthaceae \\
\hline $\begin{array}{l}\text { 221. Celaenorrhinus } \\
\text { munda munda Moore }\end{array}$ & Himalaya. & NA; LO. & SE. & $\mathrm{R}$ & 0 to 2 & M : iv- ix. & $?$ & $?$ & $?$ \\
\hline $\begin{array}{l}\text { 222. Celaenorrhinus } \\
\text { pulomaya Moore }\end{array}$ & Himalaya. & NA; LO. & SE. & $\mathrm{R}$ & 0 to 2 & ?S: iv. & $?$ & $?$ & $?$ \\
\hline $\begin{array}{l}\text { 223. Lobocla liliana } \\
\text { ignatius Plotz }\end{array}$ & W. Himalaya. & NA. & SE. & C & 0 to 2 & $B: v-v i ; x$ & $?$ & $?$ & ?Leguminosae \\
\hline $\begin{array}{l}\text { 224. Tagiades } \\
\text { litigiosa litigiosa } \\
\text { Moschler }\end{array}$ & Himalaya to China. & NA. & $\begin{array}{l}\text { SE ; } \\
\text { TD. }\end{array}$ & C & 0 to 2 & ?S : ix. & $?$ & $\mathrm{O}$ & Dioscoreaceae. \\
\hline $\begin{array}{l}\text { 225. Tagiades japetus } \\
\text { ravi Moore }\end{array}$ & $\begin{array}{l}\text { Himalaya to Indo- } \\
\text { China. }\end{array}$ & NA ; ?RM. & ?TD. & V & $?$ & M : iv-x. & $?$ & $\mathrm{O}$ & Dioscoreaceae. \\
\hline $\begin{array}{l}226 . \\
\text { Pseudocoladenia dan } \\
\text { fatih Kollar }\end{array}$ & India to Malaysia. & NA. & $\begin{array}{l}\text { SE ; } \\
\text { TD ; } \\
\text { S. }\end{array}$ & C & 0 to 2 & $B$ : iv-v; $x$ & $?$ & $\mathrm{O}$ & Amaranthaceae. \\
\hline $\begin{array}{l}\text { 227. Sarangesa } \\
\text { dasahara Moore }\end{array}$ & India to Malaysia. & NA. & $\begin{array}{l}\text { S } \\
\text { SE } \\
\text { TD. }\end{array}$ & C & 0 to 2 & M : v-xi. & $?$ & $\mathrm{O}$ & Acanthaceae \\
\hline $\begin{array}{l}\text { 228. Sarangesa } \\
\text { purendra purendra } \\
\text { Moore }\end{array}$ & India. & NA. & $\begin{array}{l}\text { S ; } \\
\text { SE ; } \\
\text { TD. }\end{array}$ & C & 0 to 2 & $\begin{array}{l}\mathrm{M}: \text { iii-vi } \\
\quad \text { ix-x }\end{array}$ & $?$ & ?M & Acanthaceae. \\
\hline $\begin{array}{l}\text { 229. Caprona alida } \\
\text { yerburyi Evans }\end{array}$ & India to China. & SM. & $?$ & V & ?2 to 3 & $M: v-x i$ & $?$ & $?$ & $?$ \\
\hline $\begin{array}{l}\text { 230. Spialia galba } \\
\text { Fabricius }\end{array}$ & India to Myanmar. & NA. & $\begin{array}{l}\mathrm{S} ; \\
\mathrm{G} \\
\mathrm{SE}\end{array}$ & $\mathrm{O}$ & 0 to 2 & $B$ : iv-v ; $x$ & $?$ & $?$ & $?$ \\
\hline $\begin{array}{l}\text { 231. Suastus gremius } \\
\text { Fabricius }\end{array}$ & India to China. & NA. & S; G. & $\mathrm{O}$ & 0 to 2 & M : vi-x. & $?$ & $?$ & $?$ \\
\hline $\begin{array}{l}\text { 232. Aeromachus } \\
\text { stigmata Moore }\end{array}$ & Himalayan & NA. & $\begin{array}{l}\text { SE; } \\
\text { S. }\end{array}$ & A & 0 to 2 & $S: v$ & $?$ & $\mathrm{O}$ & Graminae \\
\hline $\begin{array}{l}\text { 233. Notocrypta } \\
\text { feisthamelii alysos } \\
\text { Moore }\end{array}$ & India to Malaysia. & NA. & SE. & $\mathrm{O}$ & 1 to 2 & B: v; viii. & $?$ & $\mathrm{O}$ & Zingiberaceae. \\
\hline
\end{tabular}




\begin{tabular}{|c|c|c|c|c|c|c|c|c|c|}
\hline 1 & 2 & 3 & 4 & 5 & 6 & 7 & 8 & 9 & 10 \\
\hline $\begin{array}{l}\text { 234. Notocrypta } \\
\text { curvifascia Felder \& } \\
\text { Felder }\end{array}$ & India to Malaysia. & NA. & SE. & $\mathrm{O}$ & 1 to 2 & B: iv-vi. & $?$ & $\mathrm{P}$ & $\begin{array}{l}\text { Costaceae; } \\
\text { Zingiberaceae; } \\
\text { Musaceae. }\end{array}$ \\
\hline $\begin{array}{l}\text { 235. Erionota torus } \\
\text { Evans }\end{array}$ & $\begin{array}{l}\text { Himalaya to } \\
\text { Malaysia. }\end{array}$ & NA. & $\begin{array}{l}\text { SE; } \\
\text { TD. }\end{array}$ & $\mathrm{R}$ & 0 to 2 & B : iii ; ix-x & $?$ & $\mathrm{P}$ & $\begin{array}{l}\text { Musaceae; } \\
\text { Plamae }\end{array}$ \\
\hline $\begin{array}{l}\text { 236. Matapa aria } \\
\text { Moore }\end{array}$ & $\begin{array}{l}\text { India to the } \\
\text { Philippines. }\end{array}$ & SM. & ?SR. & V & $?$ & M : iii. & $?$ & $\mathrm{O}$ & Graminae. \\
\hline $\begin{array}{l}\text { 237. Taractrocera } \\
\text { danna Moore }\end{array}$ & India. & NA. & S; G. & $\mathrm{O}$ & 0 to 2 & S : v-vi. & $?$ & $?$ & ?Graminae. \\
\hline $\begin{array}{l}\text { 238. Ampittia } \\
\text { dioscorides } \\
\text { dioscorides Fabricius }\end{array}$ & India to Myanmar. & SM. & S; G. & $\mathrm{O}$ & 1 to 2 & S: viii. & $?$ & $\mathrm{O}$ & Graminae \\
\hline $\begin{array}{l}\text { 239. Udaspes folus } \\
\text { Cramer. }\end{array}$ & India to Malaysia & G; NA. & S; G. & $\mathrm{O}$ & 1 to 2 & $B$ : iv-ix & $?$ & $\mathrm{P}$ & $\begin{array}{l}\text { Zingiberaceae; } \\
\text { Liliaceae; } \\
\text { Loganiaceae. }\end{array}$ \\
\hline $\begin{array}{l}\text { 240. Pelopidas } \\
\text { assamensis Wood- } \\
\text { Mason \& de Nicéville }\end{array}$ & India to Malaysia. & NA. & SE. & $\mathrm{R}$ & 1 to 2 & M : ii-xi. & $?$ & $\mathrm{O}$ & Graminae \\
\hline $\begin{array}{l}\text { 241. Pelopidas } \\
\text { mathias Fabricius }\end{array}$ & India to China. & NA. & $\begin{array}{l}\text { SE } \\
\text { S. }\end{array}$ & $A$ & 0 to 2 & M : iii-xi. & $?$ & $\mathrm{P}$ & $\begin{array}{l}\text { Graminae, } \\
\text { Leguminosae, } \\
\text { Palmae }\end{array}$ \\
\hline $\begin{array}{l}\text { 242. Polytremis } \\
\text { lubricans Herrich- } \\
\text { Schaeffer }\end{array}$ & India to Sulawesi. & NA. & SE. & V & $?$ & B: iv; ix. & $?$ & $?$ & $?$ \\
\hline $\begin{array}{l}\text { 243. Polytremis eltola } \\
\text { Hewitson }\end{array}$ & India to Malaysia. & NA. & $\begin{array}{l}\mathrm{S} ; \\
\mathrm{SE}\end{array}$ & $\mathrm{O}$ & 0 to 2 & S : ix-x. & $?$ & $\mathrm{O}$ & Graminae. \\
\hline
\end{tabular}

permission from Mattoni (1990) and Mattoni \& Vannucci (2008), for which I am grateful.

The information recorded in Table 1 regarding voltinism is based on specimens recorded or bred, while the information regarding diapause is based on breeding experiments by Fred Smetacek Sr. and the author.

\section{WATERSHED FUNCTIONS}

Both the Bhimtal and Sattal lake systems depend largely on subsurface water from this watershed to sustain them. This fact was acknowledged by Atkinson (1882) in the words, "The hills on the western side of the [Bhimtal] lake [i.e. present day Jones Estate] are considerably higher than on the east, and are of such formation that it is highly improbable that the lake can ever dry up." Of course, back then, it was improbable that Mr. Atkinson could even conceive of the present threat of urbanization facing Bhimtal in general and Jones Estate in particular (Image 3).

Today, one lake of the system, Kuatal, has actually dried up during the last decade. With regard to Kuatal, the only thing that has changed during the past 30 years is that the Himalayan Oak forest in its catchment area in Jones Estate has been severely degraded (Image 4). In the wake of nearly unprecedented rain during July and September 2010, Kuatal filled twice for the first time in over 60 years. A photograph from around 1890 (Image 5) shows the area as it apparently used to be and Image 6 shows Kuatal when it was full in 2010. The loss of Kuatal is not recognized by any level of Government. Government maps used by the Lakes Development Authority and other Governmental departments do not even have a lake marked at the spot.

The only over-ground feeder for any of the lakes, is a small stream that rises on the Aru Plain north of Bhimtal and enters the northern end of Bhimtal after a course of roughly $3 \mathrm{~km}$, of which nearly $1 \mathrm{~km}$ is underground. This stream entirely dried up for two months between May 2012 and July 2012, for the first time in history. This is an extremely worrisome development since the prospect of Bhimtal lake becoming seasonal in the coming years is a very real probability. For the first time, too, water supply from Bhimtal Lake to Haldwani City was extended from 1530 June 2012 by the State Irrigation Department due to the lack of alternate water resources to supply the city. In addition to the subsurface springs feeding the lakes, there are two over-ground water springs on the eastern 
Table 2. Annotated list of wildlife included in the Indian Wildlife (Protection) Act 1972 and subsequent notifications recorded in Jones Estate since 1974.

\section{SCHEDULE 1}

Mammals

1) 16B Leopard (Panthera pardus): regular visitor. Jones Estate forms an integral part of the home range.

2) 17. Leopard Cat (Felis bengalensis): often seen.

3) 31A: Serow (Capricornis sumatraensis): last recorded in Jones Estate on 7 November 2011

Birds

4) 2. Cheer Pheasant (Catreus wallichi): a group of 4 sighted repeatedly during 1984 to 1986 near the highest point of the Estate, Thalla Ridge.

5) 6. Lammergeyer (Gypaetus barbatus): often seen over the Estate during the daytime.

6) 7. Large Falcons (Falco peregrinus): often seen flying about the Estate.

7) 22. Kalij Pheasant (Lophura leucomelas): at least 70 individuals in over 14 parties resident on the Estate. The number greatly increases after the breeding season.

Butterflies

8) Horaga albimacula: (=Horaga albimacula viola) found on the Estate in spring.

9) Listeria dudgeoni; (=Pamela dudgeoni): one record from March 1974.

10) Nacaduba noreia hampsoni (=Prosotas noreia hampsoni): resident population. Several favoured sites. A common butterfly in June.

11) Hypolimnas misippus: singletons recorded. A male last recorded in October 2010.

\section{SCHEDULE 2}

Mammals

1) Common Langur (Presbytis entellus): several resident parties of up to 25 individuals each.

2) Rhesus Macaque (Macaca mulatta): several resident parties totaling roughly 80 individuals which greatly increases during the monsoon months.

Butterflies

3) Dodona dipoea: recorded in Jones Estate.

4) Libythea lepita: well established, common resident.

5) Bibasis sena: resident. Rare.

6) Chliaria kina: used to be common before macaques pulled off all orchids, its larval foodplants, from trees during the late 1990 s. Now present in reduced numbers.

7) Euchrysops cnejus: common in large numbers, especially during the spring migrations and in winter.

8) Horaga onyx: well established resident in several discrete populations.

9) Lampides boeticus: swarms in spring. In smaller numbers the rest of the year.

10) Poritia hewitsoni: a few individuals of this low elevation butterfly recorded over the years.

11) Pratapa deva: one record of a male from Sattal, May 1990; another male in 1994.

11) Rapala varuna: a common, well established butterfly on the Estate.

12) Spindasis nipalicus: a common, well established species. Jones Estate holds the densest known population of this butterfly.

13) Tajuria diaeus: single record of a male, 1974.

14) Tajuria illurgis: two stable populations in Jones Estate.

15) Thecla ataxus ataxus (=Thermozephyrus ataxus ataxus): a pair recorded on the western border of the Estate in October1980.

16) Thecla ziha (=Euaspa ziha): extremely common in some years. Jones Estate holds one of three known populations of this butterfly and probably the largest one.

17) Diagora persimilis (=Hestina persimilis): the only known population of this butterfly in Uttarakhand is in Jones Estate, where it is quite common.

18) Eriboea dolon (=Polyura dolon): stragglers from higher elevation recorded.

19) Euripus consimilis: present in small numbers.

20) Hypolimnas misippus: stragglers from lower elevation stay for a few days, patrolling a beat.

21) Limenitis danava (=Auzakia danava): a resident. Small numbers regularly seen every year.

22) Neptis zaida: the only known population in Nainital district. Two other populations are known, one in the main range and one in Garhwal.

23) Pantoporia asura asura (=Athyma asura): single record from Jones Estate in October 1969.

24) Symbrenthia niphanda: small numbers recorded over the year. Apparently quite rare. Seen sporadically.

25) Metaporia agathon (=Aporia agathon) a common resident but several populations of this butterfly on the Estate have been wiped out recently due directly to destruction of its larval hostplant, Berberis sp.

26) Elymnias malelas nilamba: resident population. Common in some years. Population greatly diminished over most of the Estate due to habitat change.

27) Erebia hyagriva (=Dallacha hyagriva): common resident.

28) Mycalesis heri: a common resident species.

Mammals

29) Civets (Viverridae):Common Palm Civet (Paradoxurus hermaphroditus) a pest on cultivated fruit including mulberries.

30) Civets (Viverridae): Himalayan Palm Civet (Paguma larvata): common on cultivated fruit.

31) Flying Squirrel: Petaurista petaurista: common but eradicated from most parts of the Estate during the last 20 years due to habitat destruction

32) Himalayan Black Bear (Selenarctos thibetanus): occasional winter visitor in severe winters. Last personally seen in 1986 but there have been subsequent reports of this animal on the Estate.

33) Jackal (Canis aureus): common. At least one resident family.

34) Martens (Martes flavigula): commonly seen, especially in winter.

35) Otters (Lutra sp.): one species steals all fish from fish ponds in the area. Probably common otter.

36) Red Fox (Vulpes vulpes): regular winter visitor. Sightings usually between December to March, but one individual seen in May 2009.

37) Weasels: Himalayan yellow-bellied weasel (Mustela kathiah): a pair resident for a few months in summer 2002

Reptiles and Amphibians

38) Varanus species: Monitor Lizard: a few individuals reside on the Estate.

39) Indian Cobra (Naja naja): resident: last seen in September 2010 at the Butterfly Research Centre, Jones Estate. 


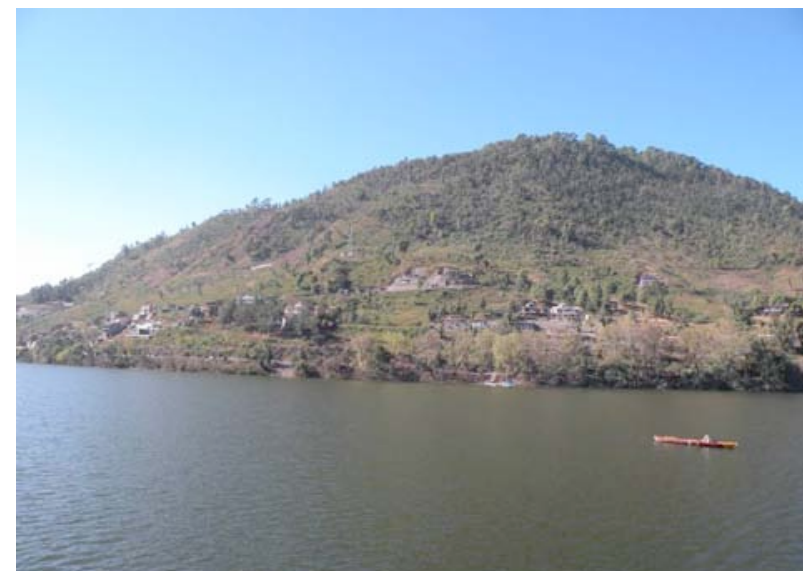

Image 3. Looking west over Bhimtal Lake, showing ongoing urbanization on Jones Estate watershed in January 2012.

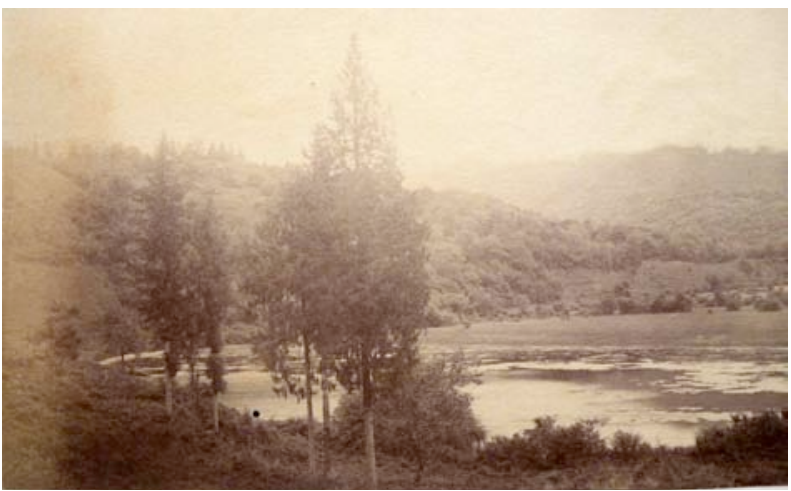

Image 5. Kuatal, circa 1890.

face of the watershed and three on its western face. In addition, there are several sub surface springs on the lake beds of both lake systems. Of the over-ground springs, both eastern face springs used to be perennial but one is now almost seasonal, with its flow reduced to a thin trickle during the dry season. Similarly, all of the three western-face springs, which used to be perennial, are seasonal today, due, presumably, to depleted Oak forest in the catchment areas of these springs. It is clear that as long as the Oak forest was in good condition, the springs were perennial. Changes to the Oak forest ecosystem in the form of the large scale drying up of trees due to forest fires, destruction of the canopy due to lopping for fodder, cutting of trees, etc were reflected in reduced flow during the dry season and increased flow, sometimes even forming new outlets, during the wet season.

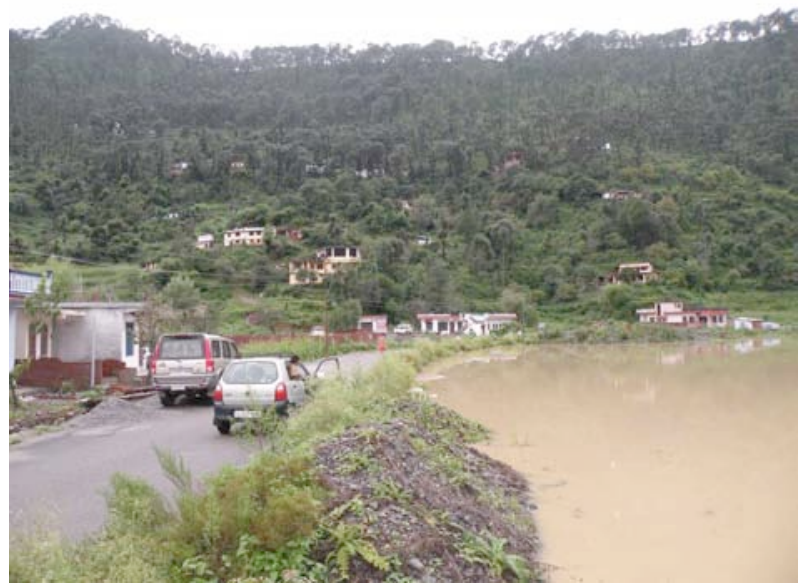

Image 4. Kuatal catchment in the background, showing degraded broadleaf forest when Kuatal filled for the first time in living memory July 2010.

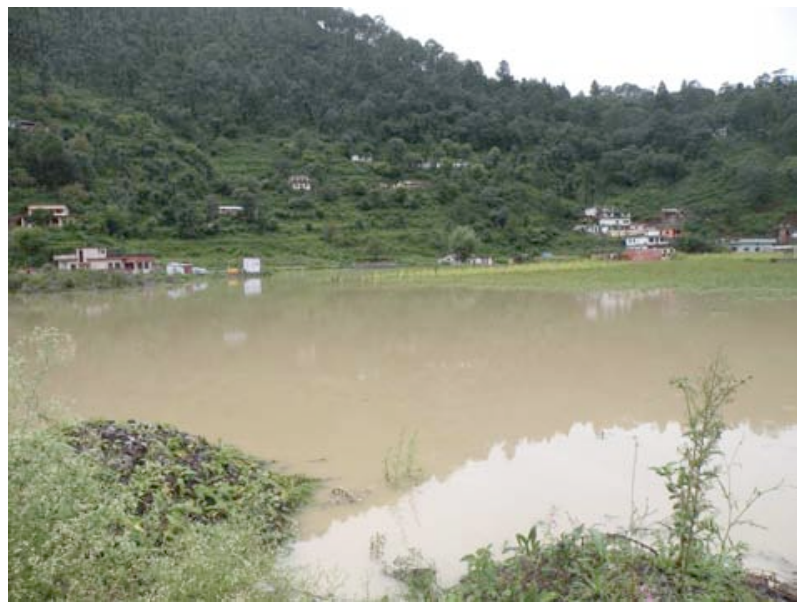

Image 6. Kuatal when it filled in July 2010 for the first time in living memory.

\section{DISCUSSION}

The Jones Estate watershed separates two lake systems which together constitute five perennial lakes and several seasonal ones. These lakes are an inestimably valuable resource of drinking quality water for future generations. However, with the urbanization of the Jones Estate watershed, their future looks uncertain, especially the Sattal lake system, which has no over-ground tributaries and a very small catchment area.

Of the $400+$ species of butterflies known from Uttarakhand (Mackinnon \& de Nicéville 1899; Hannyngton 1910, 1915; Smetacek 1987, 1992, 1993a, 1995, 1999, 2001, 2002, in press b, unpub. data; 
Singh 2003, 2005, 2005a, 2006, 2007), 243 species of butterflies (Annexure 1) and approximately 550 species of macro-moths (Smetacek 2008, 2009) have been recorded from the Jones Estate watershed. This may be compared with the entire Indian Peninsula, which is home to 330 species of butterflies (Gaonkar 1996) and an undetermined number of moth species.

Forty nine species of wildlife included in the Schedules of the Indian Wildlife Protection Act, 1972 have been recorded on the watershed (Table 2), yet even this impressive figure is not enough to prevent the ongoing destruction of this forest ecosystem.

Of special interest is the record of Lister's Hairstreak Pamela dudgeoni de Nicéville (=Listeria dudgeoni) (Lepidoptera: Lycaenidae) in March 1974 in Jones Estate. This butterfly was known from Sikkim (Type locality: specimen described in 1894) and Mussoorie (Evans 1932). Wynter-Blyth (1957) noted a specimen from Siuni camping ground near Ranikhet in Kumaon. The single specimen was recorded in 1909 and is currently in the collection of the Natural History Museum, London. It has not been recorded from Sikkim since the type was reported and was evidently recorded from Mussoorie between 1899 and 1932, since Mackinnon \& de Nicéville (1899) do not report it. The male specimen on whose basis Evans (1932) reported it from 1829m (6000ft) elevation in Mussooorie is currently in the collection of the Forest Research Institute, Dehradun. Sidhu (2011) has reported the substantial loss of habitat for the Lycaenidae in Mussoorie. It is almost certain that Pamela dudgeoni will not be found in that area now. This leaves Jones Estate as the only known habitat where this extremely rare butterfly has been recorded recently. The genus is monobasic and was believed to be confined to the Himalaya (Varshney 1997; 2010) although Inayoshi (1996-2011) has reported it from Thailand. It is not improbable that the ongoing destruction of the insect's habitat in Jones Estate will result in the global extinction of the species.

Besides this, Jones Estate is one of three known localities for Euaspa ziha de Nicéville (Lepidoptera: Lycaenidae) (P. Smetacek unpub. data); one of two known localities for Neptis miah varshneyi Smetacek; one of two known localities for Comostola hauensteini Smetacek (Lepidoptera: Geometridae) (Smetacek 2002). It supports one of the two known populations of Hestina persimilis Westwood in Uttarakhand
(Schedule 2, Indian Wildlife (Protection) Act 1972) (P. Smetacek unpub. data).

Populations of most butterfly species listed in Tables 1 and 2 are now concentrated around the Butterfly Research Centre, due to the destruction of habitat in other parts of the Estate, prior to the process of building houses. However, the area where these butterfly and moth species are now confined is very small and the possibility that they become locally extripated from the Estate in the near future is a very real probability.

The only way to have prevented the ongoing destruction and to stabilize the Bhimtal and Sattal lake systems as a drinking water resource for future generations would be, as earlier, to ban the construction of houses, resorts and cottages on the Estate. However, lacking Governmental will to do so, the future outlook for this watershed is bleak from the point of view of insect communities, water resources, the existing forest ecosystems and especially for the residents of the areas downstream, who might have relied upon the Bhimtal and Sattal lakes for a dependable supply of drinking quality water.

\section{RECOMMENDATIONS}

If competent decision making bodies make the following decisions and ensure their implementation, the gravity of the situation outlined above can be reduced or reversed in the long term:

1. Re-declare Jones Estate a Green Belt, with the area extended to include the area outlined in Fig. 1.

2. Establish a competent Central Government committee to look into the implementation of existing laws, especially the Forest Conservation Act, 1980 and the bylaws of the Lakes Development Authority, in Jones Estate, and break down illegal constructions to restore the status quo ante.

3. Ban the keeping of cattle and goats within the jurisdiction of the Bhimtal Town Area Committee in the same way this is banned within the Nainital Municipal Area.

Extrapolating the results of the above findings to the catchment area of Bhimtal and Sattal lake systems is not difficult, since the areas are contiguous and within the same altitudinal limits. 
If the Bhimtal and Sattal lakes systems are to be stabilized in the long term, then the following points may be taken into consideration:

1. An immediate ban be put into effect on construction of roads, houses and commercial establishments within the catchment areas of Bhimtal and Sattal lakes, i.e. extending from the Catholic Church in Bhowali, east along the crest line to Ghorakhal, thence along the ridges to the hamlet of Binayak, south along the crest of Karkot and thence west to Bohrakoon hamlet, then further west to Suriya Gaon and northwards along the crest of Hidamba (Hirrup) to the crest of Hatchhina and thence again following the line of crests to the Catholic Church in Bhowali. The same provisions as applied in Nainital or Mussoorie Municipalities regarding construction be made applicable within this area.

2. Within this area, so as not to alienate residents, a provision be made to permit residents for at least two generations (i.e. 40 years) to construct additional housing for extended families or build commercial establishments for a source of family income. The verification for this may be made sensible but strict so that lower officials do not consider the possibility of issuing fake reports or certificates, as the case may be.

3. A ban on cattle and goat breeding be implemented within the jurisdiction of the Bhimtal Town Area Committee, on the same lines as that in force within Nainital Cantonment and Municipality.

4. A ban on free range grazing by cattle within the catchment area, to be enforced by concerned agencies, i.e. Forest Department and Van Panchayats.

5. A wood depot be set up in Bhimtal so that residents are able to purchase fuel legally, as in other small towns throughout Kumaon.

6. If necessary, one or more fodder depot(s) be set up in the higher reaches of the catchment area, eg. in Farsoli, to cater to the needs of villages in that area and reduce dependence on lopping forest trees.

All these recommendations are well within the scope of the Government, require no extra funds and will have to be implemented in due course in the Bhimtal Valley in any case. If it is done now, then the action will come into force before the damage is irreversible, unlike Nainital and Mussoorie, where the bans came into effect after the damage had been done.

\section{REFERENCES}

Anonymous (2006). The Wildlife (Protection) Act 1972. Natraj Publishers, Dehra Dun, 235pp.

Anonymous (2010). District rainfall for last 5 years. District: Nainital. Hydromet Division. India Meteorological Department.http://www.imd.gov.in/section/hydro/ distrainfall/webrain/uttarakhand/nainital.txt. Accessed on 21 July 2010.

Atkinson, E.T. (1882). The Himalayan Districts of the North West Provinces of India. Vol. 2, Chapter 2. Zoology (Invertebrata).Government Press, Allahabad, 87-266pp.

Evans, W.H. (1932). The Identification of Indian Butterflies. 2nd Edition. Bombay Natural History Society, Bombay, $10+454 p p+32 p l s$.

Gaonkar, H. (1996). Butterflies of the Western Ghats, India including Sri Lanka. A Biodiversity Assessment of A Threatened Mountain System. Centre for Ecological Sciences, Indian Institute of Science, Bangalore; Zoological Museum, Copenhagen and the Natural History Museum, London, 89pp.

Hannyngton, F. (1910). The butterflies of Kumaon. Journal of the Bombay Natural History Society 20: 130-142; 361372.

Hannyngton, F. (1915). Kumaon Butterflies. Journal of the Bombay Natural History Society 24(1): 197.

Inayoshi, Y. (1996-2011). A Check List of Butterflies in IndoChina, page on Family Lycaenidae http://yutaka.it-n.jp/ lyc4/81565001.html (accessed on 09 February 2012).

Mackinnon, P.W. \& L. de Nicéville (1899). List of butterflies of Mussoorie in the Western Himalayas and neighbouring regions. Journal of the Bombay Natural History Society 11: 205-221, 368-389, 585-605.

Mattoni, R. (1990). Butterflies of Greater Los Angeles. Lepidoptera Research Foundation, Beverly Hills, 1-20pp.

Mattoni, R. \& N. Vannucci (2008). Garden Butterflies of Buenos Aires. Lepidoptera Research Foundation, Beverly Hills, 1-24pp.

Osmaston, A.E. (1927). A Forest Flora for Kumaon. Government Press, Allahabad, 34+605pp.

Robinson, G.S., P.R. Ackery, I.J. Kitching, G.W. Beccaloni \& L. Hernandez (2001). Hostplants of The Moth and Butterfly Caterpillars of The Oriental Region. The Natural History Museum, London and Southdene Sdn. Bht., Kuala Lumpur, 744pp.

Sidhu, A.K. (2011). Changing biodiversity scenario in the Himalayan ecosystem: Mussoorie, Uttarakhand, India as revealed by the study of blue butterflies (Lycaenidae). Journal of Threatened Taxa 3(2): 1559-1563.

Singh, A.P. (2003). Distribution range extension of bush hopper butterfly, Ampittia dioscorides Fabricius (Lepidoptera: Hesperidae) into the lower western Himalayas. Indian Forester 129(8): 1046-1048.

Singh, A.P. (2005). Initial colonization of Red Pierrot butterfly, Talicada nyseus nyseus Guerin (Lycaenidae) in the lower westernHimalayas:anindicatorofthechanging environment. 
Current Science 89: 41-42.

Singh, A.P. (2005a). Recent records on the distribution, seasonality and occurrence of Redspot butterfly, Zesius chrysomallus Hübner from the lower western Himalayas. Journal of the Bombay Natural History Society 102(2): 238-239.

Singh, A.P. (2006). Range extension of Brown Gorgon butterfly, Meandrusa gyas gyas Westwood into Kedarnath Musk Deer Reserve, Western Himalayas: A lesser known species from north-east India. Indian Forester 132(12a): 187-189.

Singh, A.P. (2007). A new butterfly species of the genus Ypthima Hübner (Nymphalidae: Satyrinae) from Garhwal Himalaya, India. Journal of the Bombay Natural History Society 104(2): 191-194.

Smetacek, P. (1987). A new type of mimicry in butterflies. Journal of the Bombay Natural History Society: 83: 471.

Smetacek, P. (1992). Record of Plebejus eversmanni (Staudinger) (Lepidoptera: Lycaenidae) from India. Journal of the Bombay Natural History Society 89: 385-386.

Smetacek, P. (1993). Towards the Re-habilitation of Indian rivers. Report submitted to Times Fellowship Council, New Delhi, 53pp.

Smetacek, P. (1993a). Neptis cartica Moore (Lepidoptera: Nymphalidae) in the U.P. Himalaya. Journal of the Bombay Natural History Society 90: 527-528.

Smetacek, P. (1994). An annotated list of the Hawkmoths (Lepidoptera: Sphingidae) of Kumaon, North India: a probable case of faunal drift. Records of the Zoological Survey of India. Occasional Paper 156: 1-55.

Smetacek, P. (1995). A new altitudinal and range record for the Copper Flash Butterfly Rapala pheretimus Hewitson (Lepidoptera: Lycaenidae). Journal of the Bombay Natural History Society 92: 127-128.

Smetacek, P. (1999). The distribution and ecology of Polyura agraria Swinhoe (Lepidoptera: Nymphalidae) in India. Journal of the Bombay Natural History Society 96: 487488.

Smetacek, P. (2001). Resolution of the controversial western limit of the range of Delias acalis Godart (Lepidoptera: Pieridae). Journal of the Bombay Natural History Society 98: 298-300.

Smetacek, P. (2002). The genus Pontia Fabricius (Lepidoptera: Pieridae) in the Kumaon Himalaya. Journal of the Bombay Natural History Society 99: 224-231.
Smetacek, P. (2004). Descriptions of new Lepidoptera from the Kumaon Himalaya. Journal of the Bombay Natural History Society 101: 269-276.

Smetacek, P. (2004). On the occurrence of Marumba cristata Butler (Lepidoptera: Sphingidae) in Shimla, Himachal Pradesh, India. Journal of the Bombay Natural History Society 101: 171-172.

Smetacek, P. (2005). The Epipleminae (Lepidoptera: Uraniidae) of the Kumaon Himalaya. Journal of the Bombay Natural History Society 102: 186-194.

Smetacek, P. (2008). Moths recorded from different elevations in Nainital district, Kumaon Himalaya, India. Bionotes 10(1): 5-15.

Smetacek, P. (2009). Additions and corrections to the list of moths recorded from different elevations in Nainital district, Kumaon Himalaya, India. Bionotes 11(4): 117-118.

Smetacek, P. (2010a). A new species of Ceryx Wallengren (Lepidoptera: Arctiidae) from the Kumaon Himalaya. Journal of Threatened Taxa 2(5): 894-895.

Smetacek, P. (2010b). Climate Change and Himalayan Lepidoptera. Abstract Book. International Workshop on Mountain Biodiversity and Impacts of Climate Change with special reference to Himalayan Biodiversity Hotspot. G.B. Pant Institute of Himalayan Environment and Development, Kosi-Katarma, Almora, 130-134pp.

Smetacek, P. (2011a). Four new Lycaenid records from the Kumaon Himalaya, India. Journal of Threatened Taxa 3(2): $1555-1558$.

Smetacek, P. (2011b). A review of West Himalayan Neptini (Lepidoptera: Nympalidae). Journal of the Lepidopterists' Society 65(3): 153-161.

Special Correspondent (2001). Permission for construction in Bhimtal's Green Belt a mistake: Government accepts onus in State Assembly.(in Hindi) Amar Ujala newspaper for January 18, 2001 Dehra Dun Edition, page 1 lead story, continued on page 2. (Bhimtal ki harit patti kshetra mein nirman ki anumati galat: Sarkar nay Vidhan Sabha mein sweekar kiya).

Varshney, R.K. (1997). Index Rhopalocera Indica Part III. Genera of Butterflies from India and neighbouring Countries [Lepidoptera: Lycaenidae]. Oriental Insects 31: 83-138.

Varshney, R.K. (2010). Genera of Indian Butterflies. Nature Books India, New Delhi, 186pp.

Wynter-Blyth, M.A. (1957). Butterflies of the Indian Region. Bombay Natural History Society, Bombay, 20+523pp. +72 pls. 